\title{
Visibility Complexes and the Baues Problem for Triangulations in the Plane*
}

\author{
P. H. Edelman and V. Reiner \\ School of Mathematics, University of Minnesota, \\ Minneapolis, MN 55455, USA \\ \{edelman,reiner\}@math.umn.edu
}

\begin{abstract}
We give a positive answer for the special case of the Generalized Baues Problem which asks whether the complex of triangulations of a point set $\mathcal{A}$ in general position in the plane has the homotopy type of a sphere. In the process, we are led to define the visibility complex for a simplicial complex $P$ whose vertices lie in $\mathcal{A}$, and prove that this visibility complex has the same homotopy type as $P$. The main technique is a variant of deletion-contraction from matroid theory, along with a new method for proving homotopy equivalence of posets which we call the nerve-flag paradigm.
\end{abstract}

\section{Introduction}

The subject of triangulations of a point set $\mathcal{A}$ in $\mathbb{R}^{d}$ has undergone a recent surge of interest, partly due to the theory of secondary polytopes and $\mathcal{A}$-discriminants and resultants defined by Gelfand et al. [GKZ], and also due to a problem raised by Baues [B] in homotopy theory. These two developments were unified by Billera and Sturmfels in their work on fiber polytopes [BS], and led Billera et al. [BKS], to formulate the Generalized Baues Problem (GBP) associated to a surjection of convex polytopes $P \rightarrow Q$. Given two polytopes $P$ and $Q$ and an affine surjection $P \rightarrow Q$, the problem asks whether a certain complex of polygonal decompositions of $Q$ induced by the faces of $P$ has the same homotopy type as the subcomplex of coherent decompositions, which are defined geometrically. This subcomplex is known to be isomorphic to the boundary complex of a convex polytope (the fiber polytope $\Sigma(P, Q)$ ) and hence is spherical.

The answer to the GBP is known to be positive when $\operatorname{dim}(Q)=1$ [B1], [BKS],

\footnotetext{
* The work of Edelman and Reiner was partially supported by NSF Grant DMS-9201490, and Mathematical Sciences Postdoctoral Research Fellowship DMS-9206371, respectively.
} 

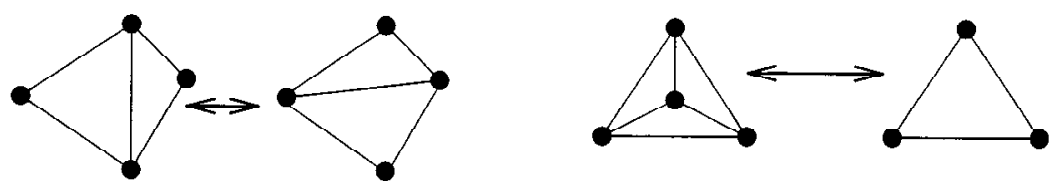

Fig. 1

and when $\operatorname{dim}(P)-\operatorname{dim}(Q) \leq 2$ [RZ], however counterexamples were given recently by Rambau and Ziegler [RZ] with $\operatorname{dim}(Q) \geq 2$ and $\operatorname{dim}(P)-\operatorname{dim}(Q) \geq 3$ (see this reference for a more complete discussion of the GBP). The most interesting cases of the GBP are those where $P$ is either an $n$-cube or an $n$-simplex, since a positive answer in these instances would resolve the weaker question of whether all cubical subdivisions of a zonotope $Z$ are connected by mutations, or all triangulations of a point set $\mathcal{A}$ are connected by bistellar operations, respectively. See [BS] for a discussion of this connection. For $P$ an $n$-cube the answer to the GBP is known to be positive for $\operatorname{dim}(Q) \leq 2$ [SZ]. For $P$ an $n$-simplex, it follows from the work of Lawson [L] that all triangulations of $\mathcal{A}$ in $\mathbb{R}^{2}$ are connected by bistellar operations, which shows that the complex considered in this case of the GBP is at least connected. For vertices in general position in $\mathbb{R}^{2}$ the bistellar operations are of two types, a diagonal flip or the insertion/removal of a vertex from the interior of a triangle. These are illustrated in Fig. 1. We note that Lawson considered only triangulations which use all vertices in $\mathcal{A}$ so that only diagonal flips were relevant.

In this paper, we will give a positive answer to the GBP for triangulations of $\mathcal{A}$ in general position in $\mathbb{R}^{2}$. In fact, we prove something stronger. For an arbitrary simplicial complex $P$ embedded in $\mathbb{R}^{2}$ and using only vertices in $\mathcal{A}$, we will show that there is a complex generalizing the complex considered by the GBP, and that when $\mathcal{A}$ is in general position in $\mathbb{R}^{2}$, this complex is contractible (Theorems 2 and 3). Our method is a variant of the deletion-contraction technique from matroid theory, in combination with a topological analysis. An interesting by-product of the analysis is that it requires us to understand the homotopy type of a simplicial complex which we call the visibility complex for the pair $(P, \mathcal{A})$, an extension of the visibility graph considered in the computational geometry literature [OR1]. We prove that this visibility complex has the same homotopy type as $P$ (Theorem 1$)$.

Perhaps a further word or two is in order about the motivation for these results. The work of [GKZ] shows that there is a large and well-behaved subset of triangulations of $\mathcal{A}$ called the regular triangulations. The graph whose vertices are these triangulations and whose edges correspond to bistellar operations forms the 1-skeleton of the $(|\mathcal{A}|-3)$ dimensional secondary polytope. It follows from Balinski's theorem [Z, §3.5] that this graph is $(|\mathcal{A}|-3)$-connected. The question asked by the Baues problem is a question of connectivity for all triangulations, not in the graph-theoretic but in the topological sense, and so may be viewed as the topological analogue of results about graph connectivity.

The paper is organized as follows. Section 2 gives the basic definitions and states the main results (Theorems 1, 2, and 3). Section 3 reduces Theorems 1 and 2 to the case where $P$ is a manifold with boundary. Section 4 completes the proof of Theorem 1, and Section 5 uses this to complete the proof of Theorem 2 . Theorem 3 is then deduced as a corollary to the proof of Theorem 2 . 


\section{Definitions and Statement of Results}

Let $\mathcal{A}$ be a finite set of points in $\mathbb{R}^{d}$. A set $P$ in $\mathbb{R}^{d}$ will be called $\mathcal{A}$-triangulable if, roughly speaking, it has a triangulation as a simplicial complex whose vertices are a subset of $\mathcal{A}$. To be more precise, let $\mathcal{A}=\left\{v_{1}, \ldots, v_{n}\right\} \subseteq \mathbb{R}^{d}$ and say that $P \subseteq \mathbb{R}^{d}$ is $\mathcal{A}$-triangulable if $\mathcal{A} \subseteq P$ and there exists an abstract simplicial complex $\Delta$ on the vertex set $\{1,2, \ldots, n\}$ (not necessarily using every index as a vertex) whose geometric realization $\|\Delta\|$ maps homeomorphically onto $P$ (endowed with the subspace topology) under the map from $\|\Delta\|$ into $\mathbb{R}^{d}$ which sends an abstract vertex $i$ to the point $v_{i}$ and is extended piecewise linearly over the simplices of $\Delta$. In what follows, we will often abuse notation by drawing no distinction among the topological space $P$, the homeomorphic space $\|\Delta\|$, and the simplicial complex $\Delta$, so that we can refer to vertices, edges in $P$, and so on.

For a set $A \subseteq \mathcal{A}$, let $\operatorname{conv}(A)$ denote the convex hull of the points of $A$. Given an $\mathcal{A}$ triangulable set $P$, define the visibility complex $\Delta_{\text {vis }}(P, \mathcal{A})$ to be the abstract simplicial complex on vertex set $\mathcal{A}$ whose simplices are the subsets $A \subset \mathcal{A}$ with $\operatorname{conv}(A) \subseteq P$. The visibility graph $G_{\text {vis }}(P, \mathcal{A})$ is the 1-skeleton (vertices and edges) of $\Delta_{\text {vis }}(P, \mathcal{A})$ (see [OR2]).

A polytopal decomposition $\delta$ of the pair $(P, \mathcal{A})$ is a set of pairs $\left\{\left(P_{i}, \mathcal{A}_{i}\right)\right\}_{i=1}^{k}$ satisfying the following conditions:

(1) For each $i$, we have $\mathcal{A}_{i} \subseteq \mathcal{A}$ and $P_{i}=\operatorname{conv}\left(\mathcal{A}_{i}\right)$.

(2) $P=\bigcup_{i=1}^{k} P_{i}$.

(3) For each $i \neq j$, the polytopes $P_{i}, P_{j}$ intersect in a common proper face $F$ of each (possibly the empty face, but not $P_{i}$ or $P_{j}$ itself), and $\mathcal{A}_{i} \cap F=\mathcal{A}_{j} \cap F$.

Note that an $\mathcal{A}$-triangulable space $P$ always has at least one polytopal decomposition coming from its $\mathcal{A}$-triangulation $\Delta$, namely $\delta=\left\{\left(P_{i}, \mathcal{A}_{i}\right)\right\}$ where $\left\{P_{i}\right\}$ are the maximal faces of $\Delta$, and $\mathcal{A}_{i}$ is the set of vertices of $P_{i}$. Furthermore, when $\mathcal{A}$ is in general position and $\delta=\left\{\left(P_{i}, \mathcal{A}_{i}\right)\right\}$ is a polytopal decomposition of $(P, \mathcal{A})$, then any union $\bigcup_{\alpha} P_{i_{\alpha}}$ is an $\left(\bigcup_{\alpha} P_{i_{\alpha}} \cap \mathcal{A}\right)$-triangulable space: simply refine each $P_{i}$ in $\delta$ to a triangulation which introduces no new vertices and restrict this simplicial complex to its faces lying in $\bigcup_{\alpha} P_{i_{\alpha}}$. We will often abuse notation and refer to $\delta$ as a polytopal decomposition of $P$ when the vertex set $\mathcal{A}$ is implicit.

Define the Baues poset $\operatorname{Baues}(P, \mathcal{A})$ to be the set of all polytopal decompositions $\delta$ of $(P, \mathcal{A})$ ordered by refinement, i.e., if

$$
\begin{aligned}
\delta & =\left\{\left(P_{i}, \mathcal{A}_{i}\right)\right\}_{i=1}^{k}, \\
\delta^{\prime} & =\left\{\left(P_{j}^{\prime}, \mathcal{A}_{j}^{\prime}\right)\right\}_{j=1}^{k^{\prime}},
\end{aligned}
$$

then $\delta \leq \delta^{\prime}$ means for each $i=1,2, \ldots, k$ there exists some $j$ so that $P_{i} \subseteq P_{j}^{\prime}$ and $\mathcal{A}_{i} \subseteq \mathcal{A}_{j}^{\prime}$.

We will prove the following results about the homotopy type of $\Delta_{\mathrm{vis}}(P, \mathcal{A})$ and $\operatorname{Baues}(P, \mathcal{A})$ (when referring to the homotopy type of a poset $Q$, we mean the homotopy type of its order complex $\Delta(Q)$; see [B2, (9.3)]). All of our results will assume that $\mathcal{A}$ is in general position in $\mathbb{R}^{2}$, i.e., no three points of $\mathcal{A}$ are collinear. It is not clear whether 


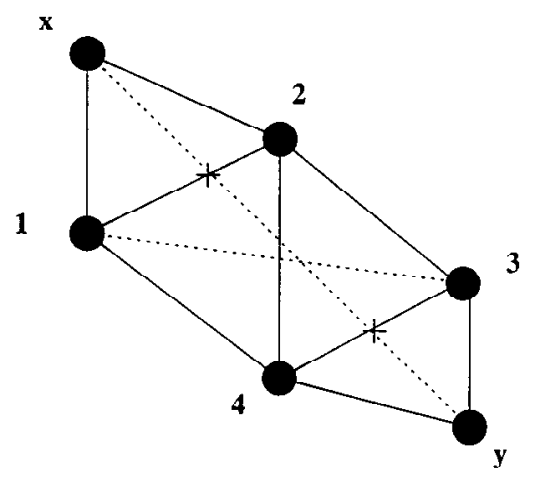

Fig. 2

this assumption is necessary for any of these results, but it will greatly simplify some of the proofs.

Theorem 1. Assume $\mathcal{A}$ is in general position in $\mathbb{R}^{2}$, and $P$ is an $\mathcal{A}$-triangulable space. Then $\Delta_{\mathrm{vis}}(P, \mathcal{A})$ is homotopy equivalent to $P$.

We note that Theorem 1 does not hold in $\mathbb{R}^{3}$ as shown by the following example. Let $1,2,3$, and 4 be vertices of a tetrahedron in $\mathbb{R}^{3}$. Let $x$ and $y$ be two points outside the tetrahedron such that the line segment joining $x$ to $y$ intersects the interior of the two edges 12 and 34 as shown in Fig. 2. If we let the space $P$ be the union of the tetrahedron 1234 and the two triangles $12 x$ and $34 y$, then $P$ is a contractible $\mathcal{A}$-triangulable space where $\mathcal{A}=\{1,2,3,4, x, y\}$. The visibility complex $\Delta_{\text {vis }}(P, \mathcal{A})$ is the complex with maximal simplices $\{1234,12 x, 34 y, x y\}$ which is easily seen to be homotopy equivalent to a circle. Although this example does not have $\mathcal{A}$ in general position, by thickening the edges 12 and 34 into triangles $122^{\prime}$ and 344' so that the edge $x y$ punctures both of these new triangles in their interiors, we obtain a general position example.

Theorem 2. Assume $\mathcal{A}$ is in general position in $\mathbb{R}^{2}$ and $P$ is an $\mathcal{A}$-triangulable space. Then $\operatorname{Baues}(P, \mathcal{A})$ is contractible.

It is easy to see that $\operatorname{Baues}(P, \mathcal{A})$ has a unique maximal element $\hat{1}$ exactly when $P=\operatorname{conv}(\mathcal{A})$. In this case $\operatorname{Baues}(P, \mathcal{A})$ is trivially contractible and the interesting problem is to describe the homotopy type of $\operatorname{Baues}(P, \mathcal{A})-\hat{1}$.

Theorem 3. Assume $\mathcal{A}$ is in general position in $\mathbb{R}^{2}$. If $P=\operatorname{conv}(\mathcal{A})$, and $\hat{1}$ is the unique top element of $\operatorname{Baues}(P, \mathcal{A})$, then $\operatorname{Baues}(P, \mathcal{A})-\hat{1}$ is homotopy equivalent to a sphere of dimension $|\mathcal{A}|-4$.

Under the hypotheses of Theorem 3, the poset $\operatorname{Baues}(P, \mathcal{A})-\hat{1}$ is isomorphic to the poset $\mathcal{S}\left(\Delta^{n-1}, P\right)$ considered in the Generalized Baues Problem [BKS, p. 554], where here $\Delta^{n-1}$ is a simplex with $n=|\mathcal{A}|$ vertices mapping onto $P$ by the canonical surjection. 
Therefore Theorem 3 resolves the instance of the GBP where $\mathcal{A}$ is in general position in $\mathbb{R}^{2}$. The case of the GBP for arbitrary point configurations $\mathcal{A}$ in $\mathbb{R}^{2}$, along with the generalizations of Theorems 2 and 3 to higher dimensions are still open.

We record here the following well-known lemma [OR1, Theorem 1.2] which is the essence of many of our later proofs:

Lemma 4. Let $C$ be an $\mathcal{A}$-triangulable Jordan curve in the plane, i.e., $C$ is a polygonal embedding of the circle $\mathbb{S}^{1} \rightarrow \mathbb{R}^{2}$ using only vertices in $\mathcal{A}$. Then the closure $\bar{C}$ of the interior of $C$ has an $\mathcal{A}$-triangulation.

It is important to note that Lemma 4 does not generalize to three dimensions. That is, there exists a nonconvex polyhedron in three dimensions whose interior cannot be partitioned into tetrahedra with vertices chosen from those of the polyhedron. See [OR1, $\S 10.2 .1]$ for a discussion of some examples.

Before closing this section, we recall a few definitions from combinatorial topology, and state a consequence of Lemma 4 , which will be used frequently later.

For $P$ an $\mathcal{A}$-triangulable space, say that $v$ in $\mathcal{A}$ is an interior vertex of $P$ if it lies in the topological interior of $P$, i.e., there is an open neighborhood $U$ of $v$ in $\mathbb{R}^{d}$ with $U \subset P$. Recall [B2, (9.9)] the link, star, and deletion of a face $F$ in a simplicial complex $\Delta$ are the subcomplexes defined by

$$
\begin{aligned}
\operatorname{link}_{\Delta}(F) & =\{G \in \Delta, G \cup F \in \Delta, G \cap F=\varnothing\}, \\
\operatorname{star}_{\Delta}(F) & =\{G \in \Delta, G \cup F \in \Delta\}, \\
\operatorname{del}_{\Delta}(F) & =\{G \in \Delta, G \cap F=\varnothing\} .
\end{aligned}
$$

Note that these definitions satisfy

$$
\begin{aligned}
\Delta & =\operatorname{star}_{\Delta}(F) \cup \operatorname{del}_{\Delta}(F), \\
\operatorname{link}_{\Delta}(F) & =\operatorname{star}_{\Delta}(F) \cap \operatorname{del}_{\Delta}(F), \\
\operatorname{star}_{\Delta}(F) & =\bar{F} * \operatorname{link}_{\Delta}(F) .
\end{aligned}
$$

where here $\bar{F}$ means the simplicial subcomplex generated by $F$, and $*$ denotes the operation of simplicial join [B2, (9.5)]. Recall that the union of two subsets of vertices in an abstract simplicial complex corresponds to the convex hull of the union of those subsets in the geometric realization.

Lemma 5. Assume $\mathcal{A}$ is in general position in $\mathbb{R}^{2}$. Let $P$ be an $\mathcal{A}$-triangulable space, $E \subseteq P$ a one-dimensional $\mathcal{A}^{\prime}$-triangulated subspace for some $\mathcal{A}^{\prime} \subseteq \mathcal{A}$, and $\mathcal{I} \subseteq \mathcal{A}$ a subset of interior vertices of $P$ which contains all the interior vertices of $P$ that lie in $E$. Then $P$ has an $\mathcal{A}$-triangulation which restricts to an $\mathcal{A}^{\prime}$-triangulation of $E$, and uses only interior vertices of $P$ which lie in $\mathcal{I}$.

Proof. Start with any $\mathcal{A}$-triangulation $\Delta$ of $P$, and convert it to one which satisfies the conclusions of the lemma in a sequence of steps. 
Step 1. If $\Delta$ uses any internal vertex $v$ of $P$ which is not in $\mathcal{I}$, then remove $v$ and all triangles containing it from $\Delta$. As $v$ is interior to $P$, the subcomplex $\operatorname{star}_{\Delta}(v)$ triangulates a 2-ball, and $\operatorname{link}_{\Delta}(v)$ triangulates a circle which is the boundary of this 2-ball. Retriangulate the interior of this 2-ball using only the vertices from $\operatorname{link}_{\Delta}(v)$ via Lemma 4. Repeat this step until all internal vertices of $P$ used in $\Delta$ lie in $\mathcal{I}$.

Step 2. If there is a vertex of $E$ which is not used in $\Delta$, then by our assumption of general position, $v$ must lie in the interior of some triangle $t$ of $\Delta$. If $t$ has vertices $v_{1}, v_{2}, v_{3}$, then remove $t$ from $\Delta$ and replace it with the three triangles $\left\{v, v_{1}, v_{2}\right\},\left\{v, v_{1}, v_{3}\right\},\left\{v, v_{2}, v_{3}\right\}$. Repeat this step until $\Delta$ uses all vertices of $E$.

Step 3. If there is an edge $e$ of $E$ which is not used in $\Delta$, let its endpoints be $v, v^{\prime}$. Consider the set of triangles in $\Delta$ which we pass through while traversing the edge $e$ from $v$ to $v^{\prime}$. By our assumption of general position, the edge $e$ crosses each of these triangles through the interiors of two of its edges, and the union of all such triangles generates a subcomplex of $\Delta$ whose boundary consists of two paths $L_{1}, L_{2}$ from $v$ to $v^{\prime}$, with the two paths separated by the edge $e$ (see Fig. 3).

Therefore if we remove all triangles and edges of $\Delta$ crossed by $e$, then $L_{1} \cup e$ and $L_{2} \cup e$ form two polygonal Jordan curves whose interiors we can triangulate by Lemma 4 using only the vertices on the curves, and now $e$ will be used in this new triangulation. Repeat this step until all edges of $E$ are used in $\Delta$.

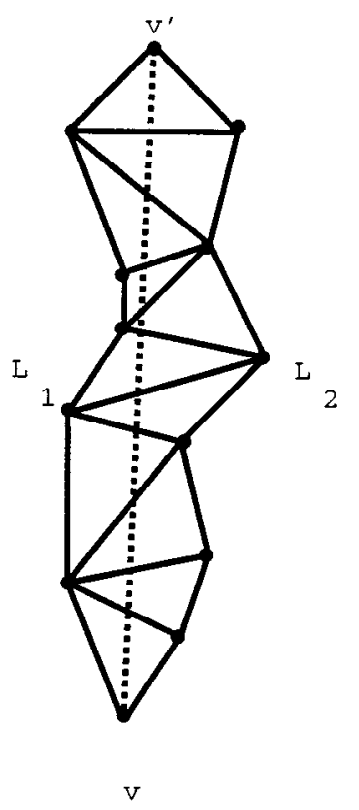

Fig. 3 


\section{Reduction to Manifold with Boundary}

In this section we simultaneously reduce via a sequence of lemmas both Theorems 1 and 2 to the case where $P$ is an $\mathcal{A}$-triangulable manifold with boundary.

The first lemma allows us to assume that $P$ (and hence $\Delta_{\text {vis }}(P, \mathcal{A})$ ) is connected.

Lemma 6. Let $P$ be an $\mathcal{A}$-triangulable set in $\mathbb{R}^{d}$, with connected components $\left\{P_{i}\right\}_{i=1}^{c}$. Then:

(1) Each $P_{i}$ is $\left(\mathcal{A} \cap P_{i}\right)$-triangulable.

(2) $\Delta_{\text {vis }}(P, \mathcal{A})$ has connected components $\left\{\Delta_{\mathrm{vis}}\left(P_{i}, \mathcal{A} \cap P_{i}\right)\right\}_{i=1}^{c}$.

(3) $\operatorname{Baues}(P, \mathcal{A})$ is isomorphic as a poset to the Cartesian product

$$
\prod_{i=1}^{c} \operatorname{Baues}\left(P_{i}, \mathcal{A} \cap P_{i}\right) .
$$

Proof. Let $\Delta$ be an $\mathcal{A}$-triangulation of $P$. Since the connected components of $\Delta$ are identified with those of $P$, the first assertion is obvious.

To prove the second assertion, we must show that $v, v^{\prime} \in \mathcal{A}$ lie in the same connected component of $P$ if and only if they lie in the same connected component of $\Delta_{\text {vis }}(P, \mathcal{A})$. But this is clear, since $v, v^{\prime}$ being connected in $P$ implies that there is a path in the 1-skeleton of $\Delta$ between them, which corresponds to a path in $\Delta_{\text {vis }}(P, \mathcal{A})$, and likewise if there is a path in $\Delta_{\text {vis }}(P, \mathcal{A})$ between them it leads to a path in $P$ between them.

To prove the third assertion, note that any decomposition $\delta$ of $(P, \mathcal{A})$ is uniquely defined by its restriction to each connected component of $P$.

The next lemma allows us to assume that the visibility graph $G_{\mathrm{vis}}(P, \mathcal{A})$ is biconnected. Recall that for a graph $G$, a vertex $v$ is an articulation point if $G-v$ is disconnected, and a graph is biconnected if it has no articulation points. Equivalently, $G$ is biconnected if any two vertices $v, v^{\prime}$ have two vertex-disjoint paths in $G$ between them. Recall also that the one-point wedge of two topological spaces $X$ and $Y$ is defined to be the quotient of their disjoint union in which a point $x \in X$ and $y \in Y$ have been identified. That is, the two spaces have been glued together at a single point from each.

Lemma 7. Let $P$ be a connected $\mathcal{A}$-triangulable set in $\mathbb{R}^{d}$. Assume $v \in \mathcal{A}$ is an articulation point of $G_{\mathrm{vis}}(P, \mathcal{A})$. Let $\left\{\mathcal{A}_{i}\right\}_{i=1}^{c}$ be the sets of vertices of the different connected components of $G_{\mathrm{vis}}(P, \mathcal{A})-v$, and let

$$
P_{i}=\bigcup_{\substack{A \subseteq \mathcal{A}_{i} \cup\{v\} \\ \operatorname{conv}(A) \subseteq P}} \operatorname{conv}(A) .
$$

Then:

(1) Each $\left\{P_{i}\right\}$ is $\mathcal{A}_{i} \cup\{v\}$-triangulable.

(2) $P$ is homeomorphic to the one-point wedge $\bigvee_{i=1}^{c} P_{i}$, with wedge point $v$. 
(3) $\Delta_{\text {vis }}(P, \mathcal{A})$ is homeomorphic to the one-point wedge

$$
\bigvee_{i=1}^{c} \Delta_{\mathrm{vis}}\left(P_{i}, \mathcal{A}_{i} \cup v\right)
$$

with wedge point $v$.

(4) $\operatorname{Baues}(P, \mathcal{A})$ is isomorphic as a poset to $\prod_{i=1}^{c} \operatorname{Baues}\left(P_{i}, \mathcal{A}_{i} \cup v\right)$.

Proof. All four assertions follow immediately from the following observation: if $A \subseteq \mathcal{A}$ has $\operatorname{conv}(A) \subseteq P$, then all the points of $A$ lie in the same biconnected component of $G_{\text {vis }}(P, \mathcal{A})$, so $A \subseteq \mathcal{A}_{i} \cup v$ for some $i$.

The next lemma allows us to assume that $P$ has no maximal edges, i.e., every edge $e$ in the triangulation of $P$ is contained in some higher-dimensional face.

Lemma 8. Let $P$ be a connected $\mathcal{A}$-triangulable set in $\mathbb{R}^{2}$. Assume $\mathcal{A}$ is in general position (no three points collinear) $G_{\mathrm{vis}}(P, \mathcal{A})$ is biconnected, and let e be a maximal edge in the triangulation $\Delta$ of $P$. Then:

(1) $P-e$ is $\mathcal{A}$-triangulable.

(2) $P$ is homotopy equivalent to the one-point wedge of the spaces $P-e$ and $a$ circle $\mathbb{S}^{1}$.

(3) $\Delta_{\mathrm{vis}}(P, \mathcal{A})$ is homotopy equivalent to the one-point wedge of the spaces $\Delta_{\mathrm{vis}}(P-e, \mathcal{A})$ and a circle $\mathbb{S}^{1}$.

(4) $\operatorname{Baues}(P, \mathcal{A})$ is isomorphic as a poset to $\operatorname{Baues}(P-e, \mathcal{A})$.

Proof. The first assertion is obvious.

The second assertion follows from the following observation: if $e$ is a maximal edge in a finite simplicial complex $\Delta$ and $\Delta-e$ is connected, then $\Delta$ is homotopy equivalent to the one-point wedge of $\Delta-e$ and $\mathbb{S}^{1}$. To see this fix one endpoint $v$ of the edge $e$, and let the other endpoint $v^{\prime}$ slide along a path connecting $v$ to $v^{\prime}$ in $\Delta-e$. This gives the homotopy between the two spaces. Note that in the situation of the lemma, $\Delta-e$ is connected since $G_{\text {vis }}(P, \mathcal{A})$ is biconnected.

The third assertion will also follow from the same observation, if we can show that the edge $\hat{e}$ in $\Delta_{\mathrm{vis}}(P, \mathcal{A})$ spanned by the endpoints $v, v^{\prime}$ of $e$ is maximal in $\Delta_{\mathrm{vis}}(P, \mathcal{A})$, since then we would have $\Delta_{\text {vis }}(P, \mathcal{A})-\hat{e}=\Delta_{\text {vis }}(P-e, \mathcal{A})$ by our general position assumption. If $\hat{e}$ were not maximal, then there exists some $v^{\prime \prime}$ so that $\operatorname{conv}\left(v, v^{\prime}, v^{\prime \prime}\right) \subseteq P$. Again by our general position assumption, $v, v^{\prime}, v^{\prime \prime}$ are not collinear, so conv $\left(v, v^{\prime}, v^{\prime \prime}\right)$ is a triangle inside $P$ which contains the edge $e=\operatorname{conv}\left(v, v^{\prime}\right)$. But this implies that some 2-simplex in $\Delta$ must contain $e$, contradicting maximality of $e$ in $\Delta$.

The fourth assertion follows from the following claim: every polytopal decomposition $\delta=\left\{P_{i}, \mathcal{A}_{i}\right\}$ of $P$ must use the edge $e$ as one of the $P_{i}$. The reason is that the union of the $P_{i}$ must cover $P$ and hence contain $e$, but $e$ is not contained in any triangle, and there are no vertices $v^{\prime \prime}$ collinear with $v, v^{\prime}$ (by our general position assumption) which could be used to subdivide $e$ or to produce a larger edge covering $e$. 
The next lemma allows us to assume that every vertex used in the triangulation $\Delta$ of $P$ has connected link.

Lemma 9. Let $P$ be a connected $\mathcal{A}$-triangulable set in $\mathbb{R}^{2}$. Assume $\mathcal{A}$ is in general position (no three points collinear) $G_{\mathrm{vis}}(P, \mathcal{A})$ is biconnected, and no edge of $P$ is maximal. Let $v$ be a vertex in the triangulation $\Delta$ of $P$ which has disconnected link. Then there exists a point set $\mathcal{A}^{\prime}$ and an $\mathcal{A}^{\prime}$-triangulable space $P^{\prime}$ such that:

(1) $P$ is homotopy equivalent to the one-point wedge of the spaces $P^{\prime}$ and a circle $\mathbb{S}^{1}$.

(2) $\Delta_{\mathrm{vis}}(P, \mathcal{A})$ is homotopy equivalent to the one-point wedge of the spaces $\Delta_{\text {vis }}\left(P^{\prime}, \mathcal{A}^{\prime}\right)$ and a circle $\mathbb{S}^{1}$.

(3) $\operatorname{Baues}(P, \mathcal{A})$ is isomorphic as a poset to $\operatorname{Baues}\left(P^{\prime}, \mathcal{A}^{\prime}\right)$.

(4) The quantity

$$
\sum_{\text {vertices } w} \hat{\beta}_{0}\left(\operatorname{link}_{\Delta}(w)\right)
$$

is smaller for $\left(P^{\prime}, \mathcal{A}^{\prime}\right)$ than for $(P, \mathcal{A})$, where $\hat{\beta}_{0}$ denotes the rank of the reduced 0-homology, which is simply one less than the number of connected components.

Proof. Construct $\mathcal{A}^{\prime}$ from $\mathcal{A}$ and $P^{\prime}$ from $P$ as in Fig. 4. To be precise, pick a connected component $C$ in $\operatorname{link}_{\Delta}(v)$, and double the vertex $v$ to create a "twin" vertex $v^{\prime}$ very near to $v$ at a small but generic distance in a generic direction toward the middle of an edge of $C$. Note that we can create such a twin by our general position assumption on $\mathcal{A}$. The connected component $C$ must contain an edge, for if $C$ were a single vertex $\{w\}$, then the edge $v w$ would be maximal in $P$. Let $\mathcal{A}^{\prime}=\mathcal{A} \cup\left\{v^{\prime}\right\}$, and obtain $P^{\prime}$ from $P$ by replacing the subcomplex $v * C$ in $\Delta$ with $v^{\prime} * C$.

It is clear that $P^{\prime}$ is $\mathcal{A}^{\prime}$-triangulated. It will be helpful in proving the rest of the assertions of the theorem to imagine an intermediate step in this process

$$
P \rightsquigarrow P^{\prime \prime} \rightsquigarrow P^{\prime},
$$

where $P^{\prime \prime}$ is obtained from $P^{\prime}$ by adding an edge from $v$ to $v^{\prime}$ as shown in Fig. 4 .

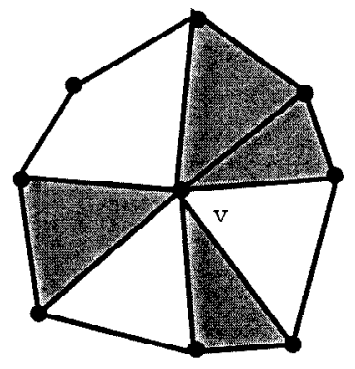

$\mathrm{p}$

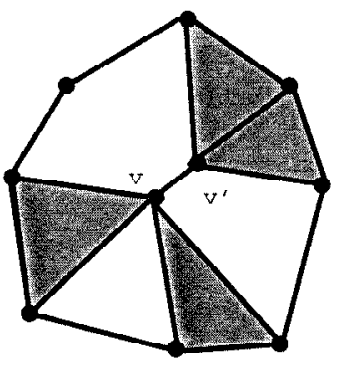

Fir

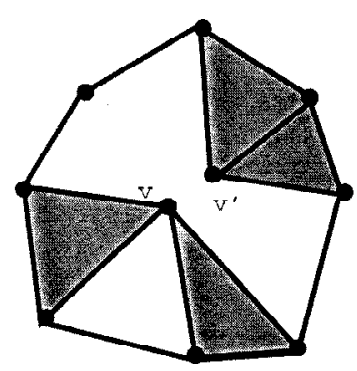

$P^{\prime}$

Fig. 4. The process $P \rightsquigarrow P^{\prime \prime} \rightsquigarrow P^{\prime}$. 
To prove the first assertion, note that $P, P^{\prime \prime}$ are obviously homotopy equivalent (shrink $v^{\prime}$ toward $v$ along the edge between them), and since $G_{\mathrm{vis}}\left(P^{\prime \prime}, \mathcal{A}^{\prime}\right)$ is still biconnected, we can apply the second assertion of the previous lemma to show that $P^{\prime}$ is homotopy equivalent to the one-point wedge of $P^{\prime \prime}$ with a circle.

To prove the second assertion, by the third assertion of the previous lemma, $\Delta_{\text {vis }}\left(P^{\prime \prime}, \mathcal{A}^{\prime}\right)$ is homotopy equivalent to the one-point wedge of $\Delta_{\text {vis }}\left(P^{\prime}, \mathcal{A}^{\prime}\right)$ with a circle. However, it is not quite as obvious that $\Delta_{\text {vis }}(P, \mathcal{A}), \Delta_{\text {vis }}\left(P^{\prime \prime}, \mathcal{A}^{\prime}\right)$ are homotopy equivalent. However this is still true, since we showed in the proof of the previous lemma that the edge $\hat{e}$ spanned by $v, v^{\prime}$ in $\Delta_{\text {vis }}\left(P^{\prime \prime}, \mathcal{A}^{\prime}\right)$ is maximal, because the edge from $v$ to $v^{\prime}$ was maximal in $P^{\prime \prime}$. Hence we can still "shrink down" $v^{\prime}$ toward $v$ in $\Delta_{\text {vis }}\left(P^{\prime \prime}, \mathcal{A}^{\prime}\right)$ along the maximal edge $\hat{e}$, and the result is $\Delta_{\text {vis }}(P, \mathcal{A})$.

The third assertion is obvious.

The fourth assertion is easy, since the number of connected components of link $\mathrm{k}_{\Delta}(w)$ is unchanged for most vertices in going from $P$ to $P^{\prime}$, is one smaller for $v$, while $v^{\prime}$ contributes 0 to the sum for $P^{\prime}$ since its link is connected.

Corollary 10. In proving Theorems 1 and 2 , it suffices to consider the case where $P$ is a connected $\mathcal{A}$-triangulable 2-manifold with boundary in $\mathbb{R}^{2}$.

Proof. By Lemmas 6-9, we may assume (using induction on various quantities) that $P$ is connected, has no maximal edges, and has the link of every vertex connected. We claim that this implies $P$ is a manifold with boundary.

To see this, choose some point $p \in P$, and we will show that it has a neighborhood homeomorphic to either $\mathbb{R}^{2}$ or the upper half-plane $\mathbb{R}_{+}^{2}$. If $p$ lies in the interior of a 2 -simplex in the $\mathcal{A}$-triangulation $\Delta$ of $P$, then it has a neighborhood in this 2 -simplex homeomorphic to $\mathbb{R}^{2}$. If $p$ lies in the interior of a 1-simplex of $\Delta$, then this 1 -simplex lies either in one or two 2-simplices, since $P$ has no maximal edges. But this then implies $p$ has a neighborhood inside these 2 -simplices homeomorphic to either $\mathbb{R}_{+}^{2}$ or $\mathbb{R}^{2}$, respectively. Lastly, if $p$ is a 0 -simplex of $\Delta$, then $\operatorname{link}_{\Delta}(v)$ is a connected one-dimensional simplicial complex, in which every vertex has degree at most 2 (else $\Delta$ would not embed in $\mathbb{R}^{2}$ ). Therefore the link is either a path or a circle, so $p$ has a neighborhood homeomorphic to either $\mathbb{R}_{+}^{2}$ or $\mathbb{R}^{2}$, respectively, inside $\operatorname{star}_{\Delta}(v)=v * \operatorname{link}_{\Delta}(v)$.

In preparation for the following two sections, we establish some notation and terminology about $(P, \mathcal{A})$ when $P$ is a connected 2-manifold with boundary in $\mathbb{R}^{2}$, and $\mathcal{A}$ is in general position.

Since $P$ is a 2-manifold with boundary embedded in $\mathbb{R}^{2}$, it follows that its boundary is a collection $\left\{C_{i}\right\}_{i=1}^{k}$ of $\mathcal{A}$-triangulable polygonal Jordan curves (which in the computational geometry literature are referred to simply as polygons), and there will be one such curve $C$ containing all the rest inside it. A vertex $v$ in $\mathcal{A} \cap C$ is called convex if its interior angle is less than $\pi$. It is well known that every polygonal Jordan curve has at least three convex vertices. 


\section{Proof of Theorem 1}

We recall here the statement of Theorem 1 .

Theorem 1. Let $\mathcal{A}$ be a finite set of points in general position in $\mathbb{R}^{2}$. Then $\Delta_{\mathrm{vis}}(P, \mathcal{A})$ is homotopy equivalent to $P$.

The proof of Theorem 1 is essentially a deletion-contraction argument, using induction on the cardinality $|\mathcal{A}|$. By Corollary 10, we may assume $P$ is a 2-manifold with boundary, and we let $v$ be a convex vertex of the curve $C$ (defined at the end of the previous section) with neighbors $v^{\prime}$ and $v^{\prime \prime}$. We define three other pairs of spaces and vertex sets

$$
\left(P_{\text {del }}, \mathcal{A}_{\text {del }}\right),\left(P_{\text {star }}, \mathcal{A}_{\text {star }}\right),\left(P_{\text {link }}, \mathcal{A}_{\text {link }}\right)
$$

as follows:

$$
\begin{aligned}
P_{\text {del }} & =\bigcup_{\substack{A \subseteq \mathcal{A}-v \\
\operatorname{conv}(A) \subseteq P}} \operatorname{conv}(A), \\
P_{\text {star }} & =\bigcup_{\substack{v \in A \subseteq \mathcal{A} \\
v \in \operatorname{con}(A) \subseteq P}} \operatorname{conv}(A), \\
P_{\text {link }} & =\bigcup_{\substack{v \in A \subseteq \mathcal{A} \\
\operatorname{conv}(A) \subseteq P}} \operatorname{conv}(A-v), \\
\mathcal{A}_{\text {del }} & =\mathcal{A}-v, \\
\mathcal{A}_{\text {star }} & =\left\{v^{\prime} \in \mathcal{A}: \operatorname{conv}\left(v, v^{\prime}\right) \subseteq P\right\}, \\
\mathcal{A}_{\text {link }} & =\mathcal{A}_{\text {star }}-v .
\end{aligned}
$$

Figure 5 illustrates these constructions.

Theorem 1 will follow from these two lemmas.

\section{Lemma 11.}

(1) $P_{\mathrm{del}}$ is an $\mathcal{A}_{\mathrm{del}}$-triangulable space.

(2) $\operatorname{del}_{\Delta_{\text {vis }}(P, \mathcal{A})}(v) \cong \Delta_{\text {vis }}\left(P_{\text {del }}, \mathcal{A}_{\text {del }}\right)$.

(3) $P_{\mathrm{del}}$ is homotopy equivalent to $P$.

\section{Lemma 12.}

(1) $P_{\text {link }}$ is an $\mathcal{A}_{\text {link }}$-triangulable space.

(2) $\operatorname{link}_{\Delta_{\text {vis }}(P, \mathcal{A})}(v) \cong \Delta_{\text {vis }}\left(P_{\text {link }}, \mathcal{A}_{\text {link }}\right)$.

(3) $P_{\text {link }}$ is contractible.

To see why Theorem 1 follows from these lemmas (and for other purposes) we will 

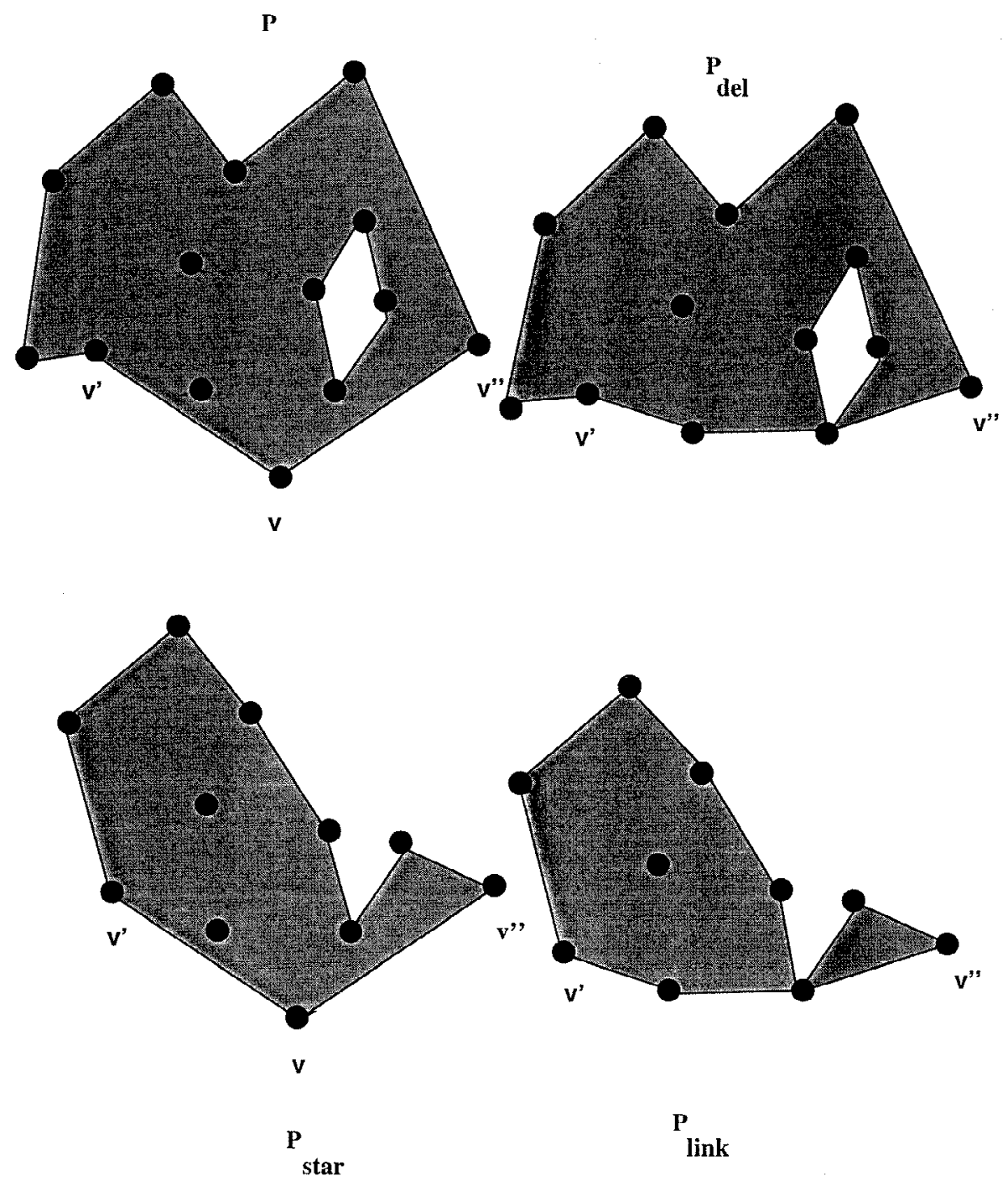

Fig. 5

make use of the following well-known fact about simplicial complexes (see, e.g., [B2, Lemma 10.3]):

Lemma 13. In a simplicial complex $\Delta$, if the link of a face $F$ is contractible, then the deletion $\operatorname{del}_{\Delta}(F)$ is a deformation retract of $\Delta$.

Using Lemma 12, assertion (2), we have that $\operatorname{link}_{\Delta_{\text {vis }}(P, \mathcal{A})}(v) \cong \Delta_{\text {vis }}\left(P_{\text {link }}, \mathcal{A}_{\text {link }}\right)$, and $\Delta_{\text {vis }}\left(P_{\text {link }}, \mathcal{A}_{\text {link }}\right)$ is homotopy equivalent to the space $P_{\text {link }}$ by induction on $|\mathcal{A}|$, so it is contractible by Lemma 12 , assertion (3). Hence $\Delta_{\text {vis }}(P, \mathcal{A})$ is homotopy equivalent 


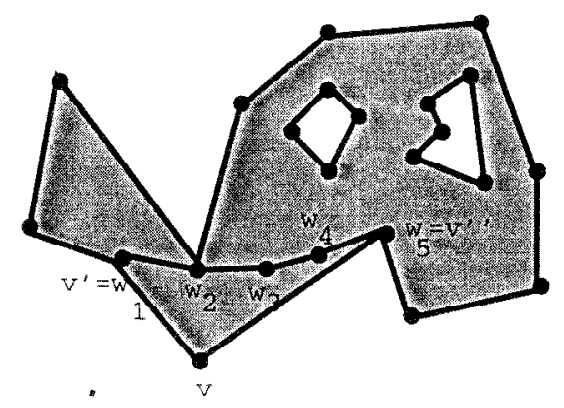

Fig. 6. The path $E$.

to $\operatorname{del}_{\Delta_{\text {vis }}(P, \mathcal{A})}(v)$ by Lemma 13 , and by Lemma 11 , assertion 2, this is isomorphic to $\Delta_{\text {vis }}\left(P_{\text {del }}, \mathcal{A}_{\text {del }}\right)$. But this is homotopy equivalent to $P_{\text {del }}$ by induction, and hence to $P$ by Lemma 11, assertion (3).

Therefore it only remains to prove Lemmas 11 and 12 .

Proof of Lemma 11. We first establish some terminology. Say that $x$ in $P$ is visible from $v$ if $\operatorname{conv}(x, v) \subseteq P$. For $x \in P_{\mathrm{del}}$, say that $v$ has an obstructed view of $x$ if $x$ is visible from $v$, but $\{x\} \neq \operatorname{conv}(v, x) \cap P_{\text {del }}$, and similarly define $v$ having an unobstructed view of $x$.

Let $E \subseteq P_{\text {del }}$ be the set

$$
E=\left\{x \in P_{\text {del }}: v \text { has an unobstructed view of } x\right\} .
$$

We claim that $E$ is a union of line segments $w_{1} w_{2}, w_{2} w_{3}, \ldots, w_{n-1} w_{n}$ in $P$ which form a path $v^{\prime}=w_{1}, w_{2}, \ldots, w_{n-1}, w_{n}=v^{\prime \prime}$ from $v^{\prime}$ to $v^{\prime \prime}$ (see Fig. 6). This claim follows from the following alternate description of the set $E$ : Let $\Delta$ be any $\mathcal{A}$-triangulation of $P$. The path $E$ is the set of points $x$ on the boundary of the polygon $\operatorname{conv}\left(\mathcal{A} \cap\left\|\operatorname{star}_{\Delta}(v)\right\|\right)$ which lie on the sides visible from $v$, i.e., $\operatorname{such}$ that $\operatorname{conv}(v, x) \cap \operatorname{conv}\left(\mathcal{A} \cap\left\|\operatorname{star}_{\Delta}(v)\right\|\right)=x$. The truth of this alternate description should be geometrically clear from Fig. 6, and we will not supply a proof, which would be tedious.

Since $E$ is a one-dimensional subspace of $P$, by Lemma 5 we can find a triangulation $\Delta$ of $P$ which restricts to a triangulation of $E$. We now claim that $\operatorname{del}_{\Delta}(v)$ triangulates $P_{\text {del }}$. To see this note that every simplex of $\operatorname{del}_{\Delta}(v)$ lies in $P_{\text {del }}$ since it lies in $P$ and does not involve the vertex $v$. Conversely, we need to show every point $x$ of $P_{\text {del }}$ lies in some simplex of $\operatorname{del}_{\Delta}(v)$. Such a point $x$ lies in some simplex $F$ of $\Delta$, and if $v$ is not a vertex of $F$, then $F$ is still a simplex of $\operatorname{del}_{\Delta}(v)$. If $v$ is a vertex of $F$, then $\operatorname{conv}(v, x)$ lies in $F$, so $\operatorname{conv}(v, x)$ must contain a point $y$ in $E$, i.e., $y$ is the closest point of $P_{\text {del }}$ to $v$ on $\operatorname{conv}(v, x)$. If $y=x$, then $x \in E \subseteq P_{\text {del }}$, and if $y \neq x$, then the simplex $F$ crosses an edge of $E$, contradicting the fact that $\Delta$ restricts to a triangulation of $E$.

From this claim, assertion (1) of Lemma 11 is now clear, and assertion (3) follows from

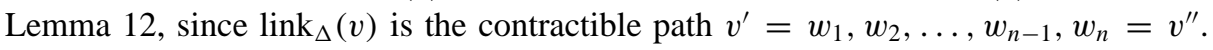
Assertion (2) is obvious once we know that $P_{\mathrm{del}}$ is $\mathcal{A}_{\mathrm{del}}$-triangulable. 
Proof of Lemma 12. Rather than showing the assertions of Lemma 12 directly, note that if we can show $P_{\text {star }}$ is an $\mathcal{A}_{\text {star }}$-triangulable contractible space, then Lemma 12 immediately follows from Lemma 11 applied to $\mathcal{A}=\mathcal{A}_{\text {star }}$ and $P=P_{\text {star }}$. Contractibility of $P_{\text {star }}$ is obvious since it is star-shaped with respect to $v$.

Therefore our strategy will be to produce an $\mathcal{A}$-triangulation $\Delta$ of $P$ which restricts to a triangulation of $\mathcal{A}_{\text {star }}$. To do this, we proceed in two steps.

Step 1. In the first step, use Lemma 5 to produce an $\mathcal{A}$-triangulation $\Delta$ of $P$ which restricts to a triangulation of the one-dimensional subspace $E \subseteq P$ defined by

$$
E=\bigcup_{\substack{x \in \mathcal{A} \\ \operatorname{conv}(v, x) \subseteq P}} \operatorname{conv}(v, x) .
$$

Step 2. Now let $\Delta^{\prime}$ be any $\mathcal{A}$-triangulation of $P$ satisfying these two properties:

(1) Every vertex $w$ in $\mathcal{A}$ which is visible from $v$ in $P$ lies inside $\left\|\operatorname{star}_{\Delta^{\prime}}(v)\right\|$.

(2) The set of vertices contained in $\operatorname{link}_{\Delta^{\prime}}(v)$ is minimal under inclusion among all triangulations satisfying (1).

We know from Step 1 that we can produce a $\Delta^{\prime}$ satisfying (1), and hence we can find one that also satisfies (2).

We now claim, that such a $\Delta^{\prime}$ has $\operatorname{star}_{\Delta^{\prime}}(v)$ triangulating $\mathcal{A}_{\text {star }}$. To prove this, note that every simplex in $\operatorname{star}_{\Delta^{\prime}}(v)$ lies in $\mathcal{A}_{\text {star }}$ by definition. Conversely, we need to show that every point $x$ of $P_{\text {star }}$ lies in some simplex of $\operatorname{star}_{\Delta^{\prime}}(v)$.

By definition such a point $x$ lies in some set $\operatorname{conv}(A) \subseteq P$ where $v \in A$ and so by Carathéodory's Theorem $x$ must lie in some triangle $\operatorname{conv}\left(v, w, w^{\prime}\right)$ where $w, w^{\prime} \in A$. Since both $w, w^{\prime}$ are visible from $v$, they lie inside $\left\|\operatorname{star}_{\Delta^{\prime}}(v)\right\|$. If $x$ also lies inside $\left\|\operatorname{star}_{\Delta^{\prime}}(v)\right\|$ we are done, so assume not and we will reach a contradiction.

It is easy to see (Fig. 7) that if $x \notin\left\|\operatorname{star}_{\Delta^{\prime}}(v)\right\|$, there must be some triple of vertices $u^{\prime}, u, u^{\prime \prime}$ which are consecutive (in this order) on the path $\operatorname{link}_{\Delta^{\prime}}(v)$, and for which $u \in \operatorname{conv}\left(v, u^{\prime}, u^{\prime \prime}\right)$. But we claim then that there are no points in $\mathcal{A} \cap \operatorname{conv}\left(u, u^{\prime}, u^{\prime \prime}\right)$, since they would be visible from $v$ but not contained in $\left\|\operatorname{star}_{\Delta^{\prime}}(v)\right\|$. Now define the one-dimensional subspace $D \subseteq P$ to be the union of the 1-skeleton of $\left\|\operatorname{star}_{\Delta^{\prime}}(v)\right\|$ and the edge conv $\left(u^{\prime}, u^{\prime \prime}\right)$, and let $\mathcal{I}$ be the set of all internal vertices of $P$ which lie in the interior of some triangle of $\left\|\operatorname{star}_{\Delta^{\prime}}(v)\right\|$. By Lemma 5, there is a triangulation of $\Delta^{\prime \prime}$ of $P$ which restricts to a triangulation of $D$, and which uses only internal vertices of $P$ in $\mathcal{I}$. The latter condition forces that $\operatorname{link}_{\Delta^{\prime \prime}}(v)=\operatorname{link}_{\Delta^{\prime}}(v)$, so that $\Delta^{\prime \prime}$ also satisfies conditions (1), (2) above. It is also true that $\Delta^{\prime \prime}$ contains the three triangles $\operatorname{conv}\left(v, u, u^{\prime}\right), \operatorname{conv}\left(v, u, u^{\prime \prime}\right), \operatorname{conv}\left(u, u^{\prime}, u^{\prime \prime}\right)$, which we can replace by the single triangle $\operatorname{conv}\left(v, u^{\prime}, u^{\prime \prime}\right)$, eliminating $u$ as a vertex in $\operatorname{link}_{\Delta^{\prime \prime}}(v)$, and contradicting condition (2) for $\Delta^{\prime \prime}$.

This completes the proof of Theorem 1 . Before closing this section, we mention a stronger statement which we were not able to prove. Note that for any $\mathcal{A}$-triangulable space $P$ in $\mathbb{R}^{d}$, there is a natural surjection

$$
\varphi:\left\|\Delta_{\text {vis }}(P, \mathcal{A})\right\| \rightarrow P \text {. }
$$




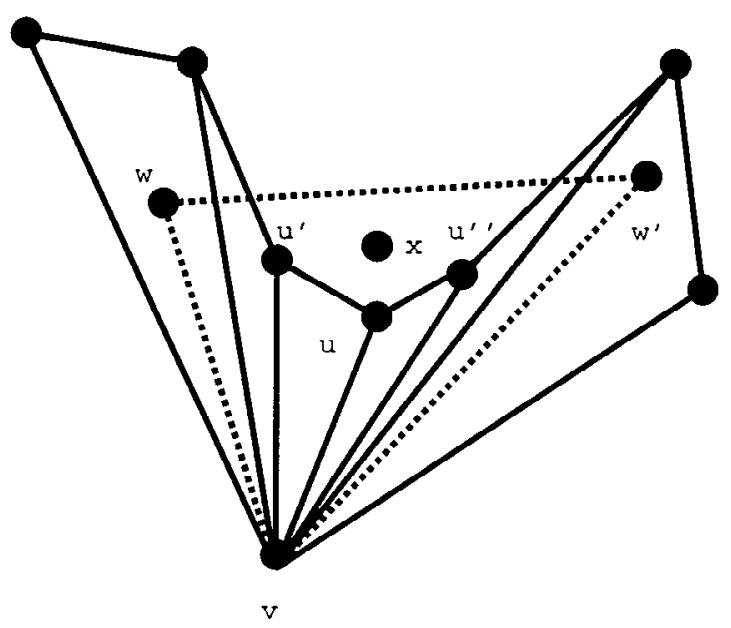

Fig. 7. Step 2 of Proof of Lemma 12.

The map $\varphi$ is defined by extending piecewise linearly over each simplex in $\Delta_{\text {vis }}(P, \mathcal{A})$ the map that sends an abstract vertex $v \in \mathcal{A}$ to the point $v \in \mathbb{R}^{d}$ which it represents.

Conjecture 14. For $\mathcal{A}$ in general position in $\mathbb{R}^{2}$, the map $\varphi$ induces the homotopy equivalence between $\Delta_{\mathrm{vis}}(P, \mathcal{A})$ and $P$.

In the case that $P=\operatorname{conv}(\mathcal{A})$, Billera and Sturmfels $[\mathrm{BS}]$ show that $\operatorname{Baues}(P, \mathcal{A})$ is a combinatorial model for the "average" fiber of $\varphi$. In our more general situation, where $P$ is an arbitrary $\mathcal{A}$-triangulable space, we might think of $\operatorname{Baues}(P, \mathcal{A})$ as still being the combinatorial model of the "average" fiber of $\varphi$. In this spirit the question of whether $\varphi$ induces a homotopy equivalence (Conjecture 14) and whether $\operatorname{Baues}(P, \mathcal{A})$ is contractible (Theorem 2) seem intimately related.

\section{Proof of Theorem 2}

In this section we prove Theorems 2 and 3, again using a variant of the deletioncontraction method along with a topological analysis. We should mention that the relation between deletion and contraction and regular triangulations of $\mathcal{A}($ when $P=\operatorname{conv}(\mathcal{A})$ ) was studied by Billera et al. [BGS] in the context of secondary polytopes. Our arguments bear a strong resemblance to the proof of Theorem 1.2 in [SZ], and to the proof of Theorem 1.2 in [BKS].

We recall here the statement of Theorem 2 .

Theorem 2. Let $\mathcal{A}$ be a finite set of points in general position in $\mathbb{R}^{2}$. Then $\operatorname{Baues}(P, \mathcal{A})$ is contractible. 
The proof of Theorem 2 uses two order-preserving maps which we will show induce homotopy equivalences

$$
\operatorname{Baues}(P, \mathcal{A}) \stackrel{f}{\rightarrow} \operatorname{Baues}_{\mathrm{vis}}(\bar{P}, \overline{\mathcal{A}}) \stackrel{g}{\rightarrow} \Delta_{\mathrm{vis}}(\hat{P}, \hat{A}),
$$

where $\operatorname{Baues}_{\text {vis }}(\bar{P}, \overline{\mathcal{A}}), \Delta_{\text {vis }}(\hat{P}, \hat{A})$ are posets which will be defined later. For both of these order-preserving maps, the fact that they induce homotopy equivalences is proven by what we call the nerve-flag paradigm and which we describe now.

\section{Nerve-Flag Paradigm}

Step 1. Define an order-preserving surjective map $h: X \rightarrow Y$ between the finite posets $X, Y$.

Step 2. Consider the covering $\mathcal{C}=\left\{C_{y}\right\}$ of $X$ by the subposets $C_{y}:=\left\langle h^{-1}(y)\right\rangle$, where for any subset $X^{\prime} \subseteq X$ the notation $\left\langle X^{\prime}\right\rangle$ means the order ideal in $X$ generated by $X^{\prime}$. Show that for any subset $Y^{\prime} \subseteq Y$, the intersection $\bigcap_{y \in Y^{\prime}} C_{y}$ is either empty or contractible.

Step 3. Let $\mathcal{N}(\mathcal{C})$ be the nerve of the cover $\mathcal{C}$ (see [B2, p. 1849]), i.e., $\mathcal{N}(\mathcal{C})$ is the simplicial complex with vertex set $Y$ and a simplex $Y^{\prime}$ whenever $\bigcap_{y \in Y^{\prime}} C_{y}$ is nonempty. Show that $\mathcal{N}(\mathcal{C})$ is actually the clique (flag) complex clique $(G)$ for a graph $G$ on the vertex set $Y$, i.e., $Y^{\prime} \subseteq Y$ forms a simplex in $\mathcal{N}(\mathcal{C})$ if and only if every pair of elements in $Y^{\prime}$ forms an edge in $G$.

Step 4. Let $G^{\prime}$ be the comparability graph of the poset $Y$, i.e., $G^{\prime}$ has vertex set $Y$, and an edge between any two elements of $y$ which are comparable in the partial order on $Y$. Show that $G^{\prime}$ is an edge-subgraph of $G$.

Step 5. Show that we can order the edges $e_{1}, \ldots, e_{s}$ in $G-G^{\prime}$ so that for $i=1,2, \ldots, s$ there exists a vertex $v_{i}$ in $G$ with the following properties:

(1) $v_{i}$ is not a vertex on the edge $e_{j}$ for $j<i$.

(2) $v_{i}$ lies below both endpoints of the edge $e_{i}$ in the partial order $Y$, and therefore forms an edge in $G^{\prime}$ with both of these endpoints.

(3) If some other vertex $w \neq v_{i}$ forms an edge in $G$ with both endpoints of edge $e_{i}$, then $v_{i}$ forms an edge with $w$ in $G$.

Lemma 15. If $h: X \rightarrow Y$ is a map of finite posets conforming to the nerve-flag paradigm, then $X$ is homotopy equivalent to $Y$.

Proof. By Step 2 and the usual Nerve Lemma [B2, Theorem 10.6], $X$ is homotopy equivalent to $\mathcal{N}(\mathcal{C})$. By Steps 3 and 4, it suffices to show that if $G^{\prime}$ is an edge-subgraph of $G$ satisfying the conditions of Step 5, then their clique complexes clique $\left(G^{\prime}\right)$, clique $(G)$ are homotopy equivalent, since clique $\left(G^{\prime}\right)$ is the same as the order complex $\Delta(Y)$.

To see this, consider the sequence of graphs $G_{0}, G_{1}, \ldots, G_{s}$ where $G_{0}=G, G_{i}=$ $G-\left\{e_{1}, \ldots, e_{i}\right\}, G_{s}=G^{\prime}$. The conditions of Step 5 imply that for $i=1,2, \ldots, s-1$, the vertex $v_{i}$ of clique $\left(G_{i}\right)$ is a cone vertex for the subcomplex $\operatorname{link}_{\operatorname{clique}\left(G_{i}\right)}\left(e_{i}\right)$ (where 
$v_{i}, e_{i}$ are both considered here as faces of $G_{i}$ ). Hence by Lemma 13 we conclude that $\operatorname{clique}\left(G_{i}\right)$ is homotopy equivalent to $\operatorname{del}_{\operatorname{clique}\left(G_{i}\right)}\left(e_{i}\right)$, which is clearly isomorphic to clique $\left(G_{i+1}\right)$. The lemma then follows by induction.

Remark. We should note that something stronger is true, namely that if $h: X \rightarrow Y$ is a map of finite posets conforming to the nerve-flag paradigm, then $h$ actually induces the homotopy equivalence between them, i.e., $h$ has a homotopy inverse. To see this, we use the Quillen Fiber Lemma [B2, Theorem 10.5] which says that $h$ induces a homotopy equivalence whenever the fibers $h^{-1}\left(Y_{\leq y}\right)$ are contractible for all $y \in Y$. When $h: X \rightarrow Y$ conforms to the nerve-flag paradigm, it is easy to check that its restrictions

$$
\left.h\right|_{h^{-1}\left(Y_{\leq y}\right)}: h^{-1}\left(Y_{\leq y}\right) \rightarrow Y_{\leq y}
$$

also conform to the nerve-flag paradigm, and hence the fibers $h^{-1}\left(Y_{\leq y}\right)$ are homotopy equivalent to their images $Y_{\leq y}$. But $Y_{\leq y}$ is always contractible since it has a top element $y$, hence the fibers are contractible and Quillen's Lemma applies.

As shown in Section 3 it suffices to prove Theorem 2 when $P$ is a 2-manifold with boundary, and we may use induction on $|\mathcal{A}|$. We let $v, v^{\prime}, v^{\prime \prime}$, and $C$ have the same meaning as in the beginning of Section 4 . Our immediate goal is to define the first of the two maps which conform to the nerve-flag paradigm.

Let $\overline{v^{\prime}}, \overline{v^{\prime \prime}}$ be points chosen close enough to $v$ on the rays $\overrightarrow{v v^{\prime}}, \overrightarrow{v v^{\prime \prime}}$, respectively, so that the triangle $\operatorname{conv}\left(v, \overline{v^{\prime}}, \overline{v^{\prime \prime}}\right)$ lies in $P$ and contains no other points of $\mathcal{A}$. Define the vertex figure $\bar{P}$ of $P$ at $v$ to be the line segment $\operatorname{conv}\left(\overline{v^{\prime}}, \overline{v^{\prime \prime}}\right)$. When $P=\operatorname{conv}(\mathcal{A})$ this notion of vertex figure coincides with the classical notion of the vertex figure of $v$ on the boundary of the polygon $P$ (see [G, p. 49]). Also define the contraction $\overline{\mathcal{A}}$ to be the collection of points $\{\bar{w}\}$ on the line segment $\bar{P}$ obtained by taking each point $w \in \mathcal{A}$ visible from $v$, and intersecting the line segment $\operatorname{conv}(v, w)$ with $\bar{P}$. Note that because of our general position assumption on $\mathcal{A}$, all the points $\{\bar{w}\}_{w \in \mathcal{A}}$ are distinct. Clearly $\bar{P}$ is an $\overline{\mathcal{A}}$-triangulable space. Therefore we have the notion of its Baues poset $\operatorname{Baues}(\bar{P}, \overline{\mathcal{A}})$.

Define a map

$$
f: \operatorname{Baues}(P, \mathcal{A}) \rightarrow \operatorname{Baues}(\bar{P}, \overline{\mathcal{A}})
$$

as follows: given $\delta=\left\{\left(P_{i}, \mathcal{A}_{i}\right)\right\} \in \operatorname{Baues}(P, \mathcal{A})$, let $f(\delta)=\left\{\left(\overline{P_{i}}, \overline{\mathcal{A}_{i}}\right): v \in P_{i}\right\}$, where $\overline{\mathcal{A}_{i}}=\left\{\bar{w}: w \in \mathcal{A}_{i}\right\}$ and $\overline{P_{i}}$ is the line segment $\operatorname{conv}\left(\overline{\mathcal{A}_{i}}\right)$. Figure 8 shows an example of the map $f$. In this example we have

$$
\begin{aligned}
\delta=\{ & \left(P_{1},\left\{v, v^{\prime}, w_{2}\right\}\right),\left(P_{2},\left\{v, w_{2}, w_{3}, w_{4}\right\}\right), \\
& \left.\left(P_{3},\left\{v, w_{4}, w_{5}\right\}\right),\left(P_{4},\left\{v, w_{5}, w_{6}, v^{\prime \prime}\right\}\right),\left(P_{5},\left\{v^{\prime}, w_{1}, w_{2}\right\}\right)\right\}, \\
f(\delta)=\{ & \left(\bar{P}_{1},\left\{\bar{v}^{\prime}, \bar{w}_{2}\right\}\right),\left(\bar{P}_{2},\left\{\bar{w}_{2}, \bar{w}_{3}, \bar{w}_{4}\right\}\right), \\
& \left.\left(\bar{P}_{3},\left\{\bar{w}_{4}, \bar{w}_{5}\right\}\right),\left(\bar{P}_{4},\left\{\bar{w}_{5}, \bar{w}_{6}, \bar{v}^{\prime \prime}\right\}\right)\right\} .
\end{aligned}
$$

Note that vertices $\bar{w}_{3}$ and $\bar{w}_{6}$ appear in $\overline{\mathcal{A}}_{2}$ and $\overline{\mathcal{A}}_{4}$, respectively, even though they are not endpoints of any of the $\bar{P}_{i}$ 's.

We wish to verify that $f$ conforms to the nerve-flag paradigm, and identify its image. It is trivial to check this map is order-preserving, so Step 1 is satisfied. 

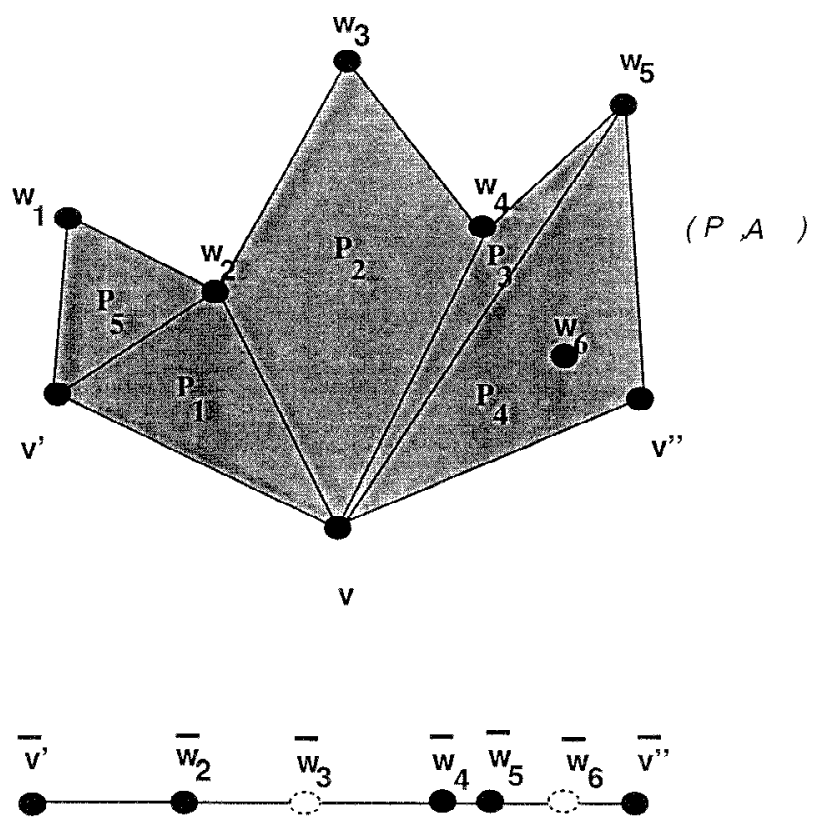

Fig. 8. An example of the map $f$.

Let Baues $_{\text {vis }}(\bar{P}, \overline{\mathcal{A}})$ be the subposet of $\operatorname{Baues}(\bar{P}, \overline{\mathcal{A}})$ consisting of those $\delta=\left\{\left(\overline{P_{i}}, \overline{\mathcal{A}_{i}}\right)\right\}$ in which each line segment $\overline{P_{i}}$ occurring satisfies

$$
\operatorname{conv}\left(\mathcal{A}_{i} \cup v\right) \subseteq P,
$$

where $\mathcal{A}_{i}$ is the set $\{w\}_{\bar{w} \in \overline{\mathcal{A}_{i}}}$. It is easy to see that this subposet is an order ideal in $\operatorname{Baues}(\bar{P}, \overline{\mathcal{A}})$, and it follows immediately from the definition of the map $f$ that its image lies in Baues vis $(\bar{P}, \overline{\mathcal{A}})$. It is not quite as obvious that this is exactly the image of $f$.

Lemma 16. The image of $f: \operatorname{Baues}(P, \mathcal{A}) \rightarrow \operatorname{Baues}(\bar{P}, \overline{\mathcal{A}})$ is $\operatorname{Baues}_{\mathrm{vis}}(\bar{P}, \overline{\mathcal{A}})$.

Proof. We must show that for any

$$
\delta^{\prime}=\left\{\left(\overline{P_{i}}, \overline{\mathcal{A}_{i}}\right)\right\}_{i=1}^{k} \in \operatorname{Baues}_{\mathrm{vis}}(\bar{P}, \overline{\mathcal{A}}),
$$

there is some $\delta \in \operatorname{Baues}(P, \mathcal{A})$ with $f(\delta)=\delta^{\prime}$. For each $i=1,2, \ldots, k$, let $\mathcal{A}_{i}$ be the set $\{w\}_{\bar{w} \in \overline{\mathcal{A}_{i}}}$ and $P_{i}=\operatorname{conv}\left(\mathcal{A}_{i}\right)$. This yields a set $\left\{\left(P_{i}, \mathcal{A}_{i}\right)\right\}_{i=1}^{k}$, and we wish to extend this set by more polygons $\left\{\left(P_{i}, \mathcal{A}_{i}\right)\right\}_{i=k+1}^{l}$ to obtain a polytopal decomposition $\delta=\left\{\left(P_{i}, \mathcal{A}_{i}\right)\right\}_{i=1}^{l}$ of $P$. If this can be done, then clearly $f(\delta)=\delta^{\prime}$.

To do this, we need only show that $P$ has an $\mathcal{A}$-triangulation $\Delta$ in which $\operatorname{star}_{\Delta}(v)$ is a triangulation of $\bigcup_{i=1}^{k} P_{i}$, since then the maximal simplices in $\operatorname{del}_{\Delta}(v)$ will give us the remaining $\left\{\left(P_{i}, \mathcal{A}_{i}\right)\right\}_{i=k+1}^{l}$ that are needed to make up $\delta$. Applying Lemma 5 in the situation where $E$ is the union of the boundary edges of the polygons $P_{i}, i=1, \ldots, k$, and $\mathcal{I}$ is the set of all interior vertices of $P$ provides such a triangulation $\Delta$. 
Having identified the image of $f$, we now proceed to verify that $f$ satisfies Steps 2 to 5 of the nerve-flag paradigm. Consider the covering $\mathcal{C}$ of $\operatorname{Baues}(P, \mathcal{A})$ by the subposets $C_{y}:=\left\langle f^{-1}(y)\right\rangle$, for $y \in \operatorname{Baues}_{\text {vis }}(\bar{P}, \overline{\mathcal{A}})$. Note that $C_{y}$ can be described geometrically as the set of all polytopal subdivisions of $P$ inducing $y$ on the vertex figure together with all decompositions below them in the $\operatorname{Baues}(P, \mathcal{A})$. We establish some notation which will prove useful. An element $y \in \operatorname{Baues}_{\mathrm{vis}}(\bar{P}, \overline{\mathcal{A}})$ is a polytopal decomposition $\left\{\left(\overline{P_{i}}, \overline{\mathcal{A}_{i}}\right)\right\}_{i=1}^{k}$, which gives rise to a set $\left\{\left(P_{i}, \mathcal{A}_{i}\right)\right\}_{i=1}^{k}$ of polygons $P_{i}$ and vertex sets $\mathcal{A}_{i}$ in $P$ as in the proof of Lemma 16. Associate to $y$ :

- the one-dimensional complex $E_{y}$ which is the union of all boundary edges of the $P_{i}, i=1, \ldots, k$

- the set of vertices $\mathcal{I}_{y}$ which is the union of all internal vertices of $\mathcal{A}$ which lie in some $\mathcal{A}_{i}, i=1, \ldots, k$; and

- the set of vertices $S_{y}$ consisting of all internal vertices of $\mathcal{A}$ that share an edge with $v$ in the boundary of some $P_{i}, i=1, \ldots, k$.

Lemma 17. The following are equivalent for a subset $Y \subseteq \operatorname{Baues}_{\mathrm{vis}}(\bar{P}, \overline{\mathcal{A}})$ :

(1)

$$
\bigcap_{y \in Y} C_{y} \neq \varnothing
$$

(2) For all $y, y^{\prime} \in Y$, we have that

$$
C_{y} \cap C_{y^{\prime}} \neq \varnothing
$$

(3) For all $y, y^{\prime} \in Y$, we have that

$$
S_{y} \cup S_{y^{\prime}} \subseteq \mathcal{I}_{y} \cap \mathcal{I}_{y^{\prime}}
$$

(4)

and $E_{y} \cup E_{y^{\prime}}$ contains no pair of edges with intersecting interiors.

$$
\bigcup_{y \in Y} S_{y} \subseteq \bigcap_{y \in Y} \mathcal{I}_{y}
$$

and $\bigcup_{y \in Y} E_{y}$ contains no pair of edges with intersecting interiors.

Proof. The implications (1) $\Rightarrow(2)$ and (3) $\Rightarrow$ (4) are trivial.

For the rest, we observe that by definition of $C_{y}$, a polytopal decomposition of $P$ is in $C_{y}$ if and only if it restricts to a polytopal decomposition of $E_{y}$ and uses only internal vertices from $\mathcal{I}_{y}$.

To deduce the implication (2) $\Rightarrow$ (3), assume there is some polytopal decomposition in $C_{y} \cap C_{y^{\prime}}$, which would have to restrict to a polytopal decomposition of $E_{y} \cup E_{y^{\prime}}$ and use only internal vertices in $\mathcal{I}_{y} \cap \mathcal{I}_{y^{\prime}}$. But this implies $E_{y} \cup E_{y^{\prime}}$ cannot have any pair of edges with intersecting interiors, and that $S_{y}, S_{y^{\prime}}$ must both lie in $\mathcal{I}_{y} \cap \mathcal{I}_{y^{\prime}}$ since they are internal vertices which lie on endpoints of edges in $E_{y} \cup E_{y^{\prime}}$.

To deduce the implication (4) $\Rightarrow(1)$, apply Lemma 5 with $E=\bigcup_{y \in Y} E_{y}$ and $I=$ $\bigcap_{y \in Y} \mathcal{I}_{y}$ to obtain a triangulation in $\cap_{y \in Y} C_{y}$. 
Lemma 18. For any subset $Y \subseteq \operatorname{Baues}_{\text {vis }}(\bar{P}, \overline{\mathcal{A}})$ the intersection $\bigcap_{y \in Y} C_{y}$ is either empty or else a finite Cartesian product of posets

$$
\prod_{\alpha} \operatorname{Baues}\left(P_{\alpha}, \mathcal{A}_{\alpha}\right)
$$

with $\mathcal{A}_{\alpha} \subsetneq \mathcal{A}$ for each $\alpha$.

Proof. Assume that $Y \subseteq \operatorname{Baues}_{\mathrm{vis}}(\bar{P}, \overline{\mathcal{A}})$ has $\bigcap_{y \in Y} C_{y}$ nonempty, and let $E=\bigcup_{y \in Y} E_{y}$. Let $\left\{P_{\alpha}\right\}$ be the topological closures of the connected components of $P-E$, and let $\mathcal{A}_{\alpha}=P_{\alpha} \cap \mathcal{I}$. By the proof of the previous lemma, any polytopal decomposition in $\bigcap_{y \in Y} C_{y}$ restricts to a decomposition of each $P_{\alpha}$, and is completely determined by these restrictions. Refine any such polytopal decomposition to a triangulation $\Delta$. Then for each $\alpha, P_{\alpha}$ is an $\mathcal{A}_{\alpha}$-triangulable space since $\Delta$ restricts to an $\mathcal{A}_{\alpha}$-triangulation of $P_{\alpha}$. This gives a poset isomorphism

$$
\bigcap_{y \in Y} C_{y} \rightarrow \prod_{\alpha} \operatorname{Baues}\left(P_{\alpha}, \mathcal{A}_{\alpha}\right)
$$

Note that since Theorem 2 is true by induction on $|\mathcal{A}|$ for each $\operatorname{Baues}\left(P_{\alpha}, \mathcal{A}_{\alpha}\right)$, and a Cartesian product of contractible posets is contractible, Lemmas 17 and 18 verify that $f$ satisfies Steps 2 and 3 of the nerve-flag paradigm. Lemma 17 also allows us to describe the graph $G$ for which the nerve $\mathcal{N}(\mathcal{C})$ is clique $(G)$ : two elements $y, y^{\prime} \in \operatorname{Baues}_{\text {vis }}(\bar{P}, \overline{\mathcal{A}})$ share an edge in $G$ if and only if $E_{y} \cup E_{y^{\prime}}$ contains no pair of edges with intersecting interiors, and $S_{y} \cup S_{y^{\prime}} \subseteq \mathcal{I}_{y} \cap \mathcal{I}_{y}^{\prime}$.

To verify Step 4 , given $y<y^{\prime}$ in $\operatorname{Baues}_{\text {vis }}(\bar{P}, \overline{\mathcal{A}})$, note that $S_{y^{\prime}} \subseteq S_{y}$ and $\mathcal{I}_{y} \subseteq \mathcal{I}_{y^{\prime}}$ so that

$$
S_{y} \cup S_{y^{\prime}}=S_{y} \subseteq \mathcal{I}_{y}=\mathcal{I}_{y} \cap \mathcal{I}_{y^{\prime}},
$$

and it is easy to check that $E_{y} \cup E_{y^{\prime}}$ can have no pair of edges with intersecting interiors. Therefore every edge of the comparability graph $G^{\prime}$ of $\operatorname{Baues}_{\text {vis }}(\bar{P}, \overline{\mathcal{A}})$ is also an edge in $G$.

To verify Step 5, we will make use of the well-known fact (see [BKS, Example 3.1]) that the poset $\operatorname{Baues}(\bar{P}, \overline{\mathcal{A}})$ is actually a lattice, in fact the lattice of faces of an $(|\mathcal{A}|-2)$ cube. As a consequence, the order ideal $\operatorname{Baues}_{\text {vis }}(\bar{P}, \overline{\mathcal{A}})$ is a meet-semilattice, i.e., there is a greatest lower bound $y \wedge y^{\prime}$ for any $y, y^{\prime}$. Now given $y, y^{\prime}$ forming an edge $e_{i}$ of $G-G^{\prime}$, let $v_{i}$ be the vertex corresponding to the poset element $y \wedge y^{\prime}$, which clearly lies below both $y, y^{\prime}$ in $\operatorname{Baues}_{\mathrm{vis}}(\bar{P}, \overline{\mathcal{A}})$. We can check that

$$
\begin{aligned}
& S_{y \wedge y^{\prime}}=S_{y} \cup S_{y^{\prime}}, \\
& \mathcal{I}_{y \wedge y^{\prime}}=\mathcal{I}_{y} \cap \mathcal{I}_{y^{\prime}}, \\
& E_{y \wedge y^{\prime}} \subseteq E_{y} \cup E_{y^{\prime}} .
\end{aligned}
$$

Given any other element $w \in \operatorname{Baues}_{\text {vis }}(\bar{P}, \overline{\mathcal{A}})$ that forms an edge of $G$ with both $y, y^{\prime}$, we conclude that

$$
S_{w} \cup S_{y} \cup S_{y^{\prime}} \subseteq \mathcal{I}_{w} \cap \mathcal{I}_{y} \cap \mathcal{I}_{y^{\prime}}
$$


and hence

$$
S_{w} \cup S_{y \wedge y^{\prime}} \subseteq \mathcal{I}_{w} \cap \mathcal{I}_{y \wedge y^{\prime}}
$$

Furthermore, $E_{y \wedge y^{\prime}} \cup E_{w}$ can contain no pair of edges with intersecting interiors since $E_{y} \cup E_{y^{\prime}} \cup E_{w}$ does not. Hence $v_{i}=y \wedge y^{\prime}$ forms an edge of $G$ with $w$, as desired. Lastly, it is possible to order the edges $e_{1}, \ldots, e_{s}$ in $G-G^{\prime}$ so that $v_{i}$ is not an endpoint of $e_{j}$ for $j<i$ as follows: If $e_{i}$ has endpoints $\left\{y_{i}, y_{i}^{\prime}\right\}$ then choose the indexing so that

$$
y_{1} \wedge y_{1}^{\prime}, y_{2} \wedge y_{2}^{\prime}, \ldots
$$

is a linear extension of the partial order dual to $\operatorname{Baues}_{\mathrm{vis}}(\bar{P}, \overline{\mathcal{A}})$, i.e., $y_{i} \wedge y_{i}^{\prime}>y_{j} \wedge y_{j}^{\prime}$ implies $i<j$. This implies that if $v_{i}$ is an endpoint of $e_{j}=\left\{y_{j}, y_{j}^{\prime}\right\}$, then $y_{i} \wedge y_{i}^{\prime}=v_{i}>$ $y_{j} \wedge y_{j}^{\prime}$, so $i<j$.

This completes the verification that $f$ conforms to the nerve-flag paradigm, and hence $f$ induces a homotopy equivalence $\operatorname{Baues}(P, \mathcal{A}) \rightarrow \operatorname{Baues}_{\text {vis }}(\bar{P}, \overline{\mathcal{A}})$ by Lemma 15 .

Our next goal is to define the second map to which we will apply the nerve-flag paradigm. Define a map

$$
g: \operatorname{Baues}_{\mathrm{vis}}(\bar{P}, \overline{\mathcal{A}}) \rightarrow 2^{\mathcal{A}}
$$

as follows: if $\delta=\left\{\left(\overline{P_{i}}, \overline{\mathcal{A}_{i}}\right)\right\}_{i=1}^{k} \in \operatorname{Baues}_{\text {vis }}(\bar{P}, \overline{\mathcal{A}})$ then there is a unique line segment $\overline{P_{1}}$ containing the vertex $\overline{v^{\prime}} \in \bar{P}$, and we set

$$
g(\delta)=\left\{w \in \mathcal{A}: \bar{w} \in \overline{\mathcal{A}_{1}}, w \neq v^{\prime}\right\} .
$$

It is easy to check this map is order-preserving, if we order $2^{\mathcal{A}}$ by inclusion of sets. Figure 9 shows an example of this map.

Let $(\tilde{P}, \tilde{\mathcal{A}})$ denote the pair obtained from $(P, \mathcal{A})$ by doing the $\left(P_{\text {link }}, \mathcal{A}_{\text {link }}\right)$ construction with respect to $v$. Then, let $(\hat{P}, \hat{\mathcal{A}})$ denote the pair obtained from $(\tilde{P}, \tilde{\mathcal{A}})$ by doing the $\left(\tilde{P}_{\text {link }}, \tilde{\mathcal{A}}_{\text {link }}\right)$ construction with respect to $v^{\prime}$ (since we can easily check that $v^{\prime}$ must be a convex vertex of the boundary curve of $\tilde{P}$.) Notice that the sets $A$ which are in the image of $g$ must have the property that $\operatorname{conv}\left(A \cup\left\{v, v^{\prime}\right\}\right) \subseteq P$. Therefore the image of

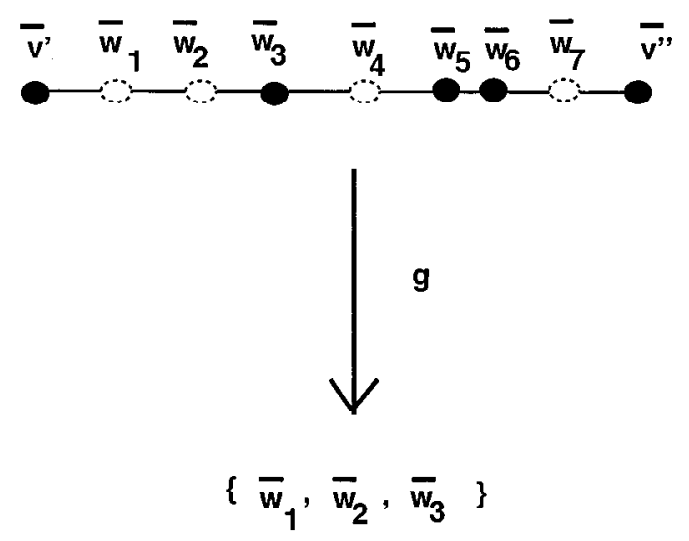

Fig. 9. An example of the map $g$. 
the map $g$ lies in the visibility complex $\Delta_{\text {vis }}(\hat{P}, \hat{\mathcal{A}})$ (thought of as its poset of faces). In fact, this characterizes the image:

Lemma 19. The image of $g$ is $\Delta_{\mathrm{vis}}(\hat{P}, \hat{\mathcal{A}})$.

Proof. By the previous remarks, we need to show that any set $A \subseteq \mathcal{A}-\left\{v, v^{\prime}\right\}$ for which conv $\left(A \cup\left\{v, v^{\prime}\right\}\right) \subseteq P$ is in the image of $g$. Tracing this back through the definition of $g$, we need to show there is a $\delta^{\prime} \in \operatorname{Baues}(\bar{P}, \overline{\mathcal{A}})$ having $\operatorname{conv}(\bar{A})$ as one of its line segments, which is equivalent by Lemma 16 to showing there is a $\delta \in \operatorname{Baues}(P, \mathcal{A})$ having conv $\left(A \cup\left\{v, v^{\prime}\right\}\right)$ as one of its polygons. If we let $E$ be the boundary complex of $\operatorname{conv}\left(A \cup\left\{v, v^{\prime}\right\}\right)$ and $\mathcal{I}$ the set of all internal vertices of $\mathcal{A}$, then Lemma 5 produces an $\mathcal{A}$-triangulation $\Delta$ of $P$ which restricts to a triangulation of $\operatorname{conv}\left(A \cup\left\{v, v^{\prime}\right\}\right)$. "Erasing" all the vertices and edges of $\Delta$ which intersect the interior of $\operatorname{conv}\left(A \cup\left\{v, v^{\prime}\right\}\right)$ gives a polytopal decomposition $\delta$ that does what we want.

Since $\hat{P}$ comes from applying the $P \rightsquigarrow P_{\text {link }}$ construction, it is contractible by Lemma 11 , and hence $\Delta_{\text {vis }}(\hat{P}, \hat{\mathcal{A}})$ is contractible by Theorem 1 . Therefore the contractibility of Baues $_{\text {vis }}(\bar{P}, \overline{\mathcal{A}})$ will follow if we can show that $g$ satisfies Steps 2 to 5 of the nerve-flag paradigm. As before, we make use of induction on the cardinality $|\mathcal{A}|$, i.e., assume that Baues $_{\text {vis }}\left(\overline{P^{\prime}}, \overline{\mathcal{A}^{\prime}}\right)$ is contractible for all $\mathcal{A}^{\prime}$-triangulated spaces $P^{\prime}$ which have a vertex $v$ where the vertex-figure $\overline{P^{\prime}}$ makes sense and $\left|\mathcal{A}^{\prime}\right|<|\mathcal{A}|$.

Consider the covering $\mathcal{C}$ of $\operatorname{Baues}_{\mathrm{vis}}(\bar{P}, \overline{\mathcal{A}})$ by the subposets $C_{A}:=\left\langle g^{-1}(A)\right\rangle$, for $A$ a face of $\Delta_{\text {vis }}(\hat{P}, \hat{\mathcal{A}})$. Linearly order $\hat{\mathcal{A}}$ by saying $w<w^{\prime}$ if $\bar{w}$ lies between $\overline{v^{\prime}}$ and $\overline{w^{\prime}}$ on the line segment $\bar{P}$. For any subset $A \subseteq \hat{\mathcal{A}}$, let $\max (A)$ denote the maximum element of $A$ in this linear order.

Lemma 20. The following are equivalent for a collection $Y$ of faces of $\Delta_{\mathrm{vis}}(\hat{P}, \hat{\mathcal{A}})$.

$$
\bigcap_{A \in Y} C_{A} \neq \varnothing
$$

(2) For all $A, A^{\prime} \in Y$, we have that

$$
C_{A} \cap C_{A^{\prime}} \neq \varnothing
$$

(3) For all $A, A^{\prime} \in Y$, we have that either $\max (A) \in A^{\prime}$ or $\max \left(A^{\prime}\right) \in A$.

(4) It is possible to linearly order the sets $A_{i}$ in $Y$ so that for all $i<j$, we have $\max \left(A_{i}\right) \in A_{j}$.

Proof. The implications (1) $\Rightarrow(2)$ and (3) $\Rightarrow$ (4) are trivial.

For the rest, we observe that by definition of $C_{A}$, a polytopal decomposition $\bar{\delta}=$ $\left\{\left(\overline{P_{i}}, \overline{\mathcal{A}_{i}}\right)\right.$ in $\operatorname{Baues}_{\mathrm{vis}}(\bar{P}, \overline{\mathcal{A}})$ will lie in $C_{A}$ if and only if $\overline{\max (A)}$ occurs as the endpoint of one of the line segments $\overline{P_{i}}$, and all the other vertices in $\bigcup_{i} \overline{\mathcal{A}_{i}}$ lying between $\overline{v^{\prime}}$ and $\overline{\max (A)}$ must be elements of $\bar{A}$. 
To deduce the implication (2) $\Rightarrow$ (3), assume there is some polytopal decomposition in $C_{A} \cap C_{A^{\prime}}$, and assume without loss of generality that $\max (A) \leq \max \left(A^{\prime}\right)$ in the linear order. But then the previous description of $C_{A}$ implies $\max (A) \in A^{\prime}$.

To deduce the implication (4) $\Rightarrow(1)$, let $\bar{\delta}$ be the triangulation of $\bar{P}$ which uses exactly the internal vertices $\{\overline{\max (A)}\}_{A \in Y}$. This triangulation is an element of $\bigcap_{A \in Y} C_{A}$.

Lemma 21. For any collection $Y$ of faces of $\Delta_{\mathrm{vis}}(\hat{P}, \hat{\mathcal{A}})$ the intersection $\bigcap_{A \in Y} C_{y}$ is either empty or else a finite Cartesian product of posets

$$
\prod_{\alpha} \text { Baues }_{\mathrm{vis}}\left(\overline{P_{\alpha}}, \overline{\mathcal{A}_{\alpha}}\right) \text {, }
$$

where for each $\alpha, P_{\alpha}$ is an $\mathcal{A}_{\alpha}$-triangulated space for which the vertex-figure $\overline{P_{\alpha}}$ makes sense, and $\mathcal{A}_{\alpha} \subsetneq \mathcal{A}$.

Proof. Assume that $\bigcap_{A \in Y} C_{A}$ is nonempty, and let $E$ be the set of vertices $\{\overline{\max (A)}\}_{A \in Y}$. Let $\left\{\overline{P_{\alpha}}\right\}$ be the line segments which are the topological closures of the connected components of $\bar{P}-E$. For each $\alpha$ let $\overline{\mathcal{A}_{\alpha}}$ be the intersection of the elements of $\bar{A}$ as $A$ ranges over those sets in $Y$ for which $P_{\alpha}$ lies between $\overline{\max (A)}$ and $\overline{v^{\prime}}$. By the proof of the previous lemma, any polytopal decomposition in $\bigcap_{A \in Y} C_{A}$ restricts to a decomposition of each $\overline{P_{\alpha}}$, and is completely determined by these restrictions. Furthermore, we can check that for each $\alpha, \overline{P_{\alpha}}$ is the vertex-figure of an $\mathcal{A}_{\alpha}$-triangulable space $P_{\alpha}$. This gives a poset isomorphism

$$
\bigcap_{A \in Y} C_{A} \rightarrow \prod_{\alpha} \text { Baues }_{\mathrm{vis}}\left(\overline{P_{\alpha}}, \overline{\mathcal{A}_{\alpha}}\right) .
$$

Note that since each Baues $_{\text {vis }}\left(\overline{P_{\alpha}}, \overline{\mathcal{A}_{\alpha}}\right)$ is contractible by induction on $|\mathcal{A}|$, Lemmas 20 and 21 verify that $g$ satisfies Steps 2 and 3 of the nerve-flag paradigm. Lemma 20 also allows us to describe the graph $G$ for which the nerve $\mathcal{N}(\mathcal{C})$ is clique $(G)$ : two elements $A, A^{\prime}$ in the poset of faces of $\Delta_{\text {vis }}(\hat{P}, \hat{\mathcal{A}})$ share an edge in $G$ if and only if either $\max (A) \in A^{\prime}$ or $\max \left(A^{\prime}\right) \in A$.

To verify Step 4 , given $A<A^{\prime}$ in the poset of faces of $\Delta_{\text {vis }}(\hat{P}, \hat{\mathcal{A}})$, we certainly have $\max (A) \in A^{\prime}$ since $A \subseteq A^{\prime}$. Therefore every edge of the comparability graph $G^{\prime}$ of $\operatorname{Baues}_{\mathrm{vis}}(\bar{P}, \overline{\mathcal{A}})$ is also an edge in $G$.

To verify Step 5, given $A, A^{\prime}$ forming an edge $e_{i}$ of $G-G^{\prime}$, let $v_{i}$ be the vertex corresponding to the face $A \cap A^{\prime}$, so clearly $v_{i}$ lies below both $A, A^{\prime}$ in the poset of faces of $\Delta_{\text {vis }}(\hat{P}, \hat{\mathcal{A}})$. Given any other face $B$ of $\Delta_{\text {vis }}(\hat{P}, \hat{\mathcal{A}})$ that forms an edge of $G$ with both $A, A^{\prime}$, we must check that $B$ forms an edge of $G$ with $A \cap A^{\prime}$. To do this, we may assume without loss of generality that $\max (A) \in A^{\prime}$, and check two cases depending upon whether $\max (B)<\max (A)$ or $\max (B) \geq \max (A)$. In the former case, we must have $\max (B) \in A, A^{\prime}$ and hence $\max (B) \in A \cap A^{\prime}$, so we are done. In the latter case, $\max (A) \in A^{\prime}, B$ and hence $\max \left(A \cap A^{\prime}\right)=\max (A) \in B$, so we are again done.

Lastly, it is possible to order the edges $e_{1}, \ldots, e_{s}$ in $G-G^{\prime}$ so that $v_{i}$ is not an endpoint of $e_{j}$ for $j<i$ as follows: If $e_{i}$ has endpoints $\left\{A_{i}, A_{i}^{\prime}\right\}$, then choose the indexing so that

$$
\left|A_{1} \cap A_{1}^{\prime}\right| \geq\left|A_{2} \cap A_{2}^{\prime}\right| \geq \cdots .
$$


This implies that if $v_{i}$ is an endpoint of $e_{j}=\left\{A_{j}, A_{j}^{\prime}\right\}$, then $A_{i} \cap A_{i}^{\prime}=v_{i} \in\left\{A_{j}, A_{j}^{\prime}\right\}$, so $\left|A_{i} \cap A_{i}^{\prime}\right|>\left|A_{j} \cap A_{j}^{\prime}\right|$ which implies $i<j$.

This completes the verification that $g$ conforms to the nerve-flag paradigm, and hence induces a homotopy equivalence $\operatorname{Baues}_{\mathrm{vis}}(\bar{P}, \overline{\mathcal{A}}) \rightarrow \Delta_{\mathrm{vis}}(\hat{P}, \hat{\mathcal{A}})$ by Lemma 15 . Since $\Delta_{\text {vis }}(\hat{P}, \hat{\mathcal{A}})$ is contractible, the proof of Theorem 2 is now complete.

From the proof of Theorem 2 we now deduce Theorem 3.

Theorem 3. Assume $\mathcal{A}$ is in general position in $\mathbb{R}^{2}$. If $P=\operatorname{conv}(\mathcal{A})$, then $\operatorname{Baues}(P, \mathcal{A})$ has a unique top element $\hat{1}$, and $\operatorname{Baues}(P, \mathcal{A})-\hat{1}$ is homotopy equivalent to a sphere of dimension $|\mathcal{A}|-4$.

Proof. When $P$ is convex, i.e., $P=\operatorname{conv}(\mathcal{A})$, the map $f: \operatorname{Baues}(P, \mathcal{A}) \rightarrow \operatorname{Baues}(\bar{P}, \overline{\mathcal{A}})$ is a surjection since $\operatorname{Baues}(\bar{P}, \overline{\mathcal{A}})=\operatorname{Baues}_{\mathrm{vis}}(\bar{P}, \overline{\mathcal{A}})$. Furthermore, the top element $1 \mathrm{i}$ $\operatorname{Baues}(P, \mathcal{A})$ is the polytopal decomposition $\{(P, \mathcal{A})\}$, having a single polygon, and this is the unique pre-image of the top element $\overline{\hat{1}}$ of $\operatorname{Baues}(\bar{P}, \overline{\mathcal{A}})$ consisting of the single pair $\{(\bar{P}, \overline{\mathcal{A}})\}$. Therefore $\operatorname{Baues}(P, \mathcal{A})-\hat{1}$ surjects onto $\operatorname{Baues}(\bar{P}, \overline{\mathcal{A}})-\overline{\hat{1}}$. Furthermore, this restricted map also conforms to the nerve-flag paradigm since $f$ did, and hence induces a homotopy equivalence. But $\operatorname{Baues}(\bar{P}, \overline{\mathcal{A}})-\overline{\hat{1}}$ is the face poset of the boundary of an $(|\overline{\mathcal{A}}|-2)$-cube, and hence triangulates a $(|\overline{\mathcal{A}}|-3)$-sphere. Since $|\overline{\mathcal{A}}|=|\mathcal{A}|-1$, Theorem 3 follows.

\section{Acknowledgments}

The authors would like to thank G. Ziegler for pointing out a serious flaw in the proof of Theorem 2 in an earlier version of this paper, which necessitated the introduction of the nerve-flag paradigm.

\section{References}

[B] H. J. Baues, Geometry of loop spaces and the cobar construction, Mem. Amer. Math. Soc., 25 (1980), 230.

[B1] A. Björner, Essential chains and homotopy type of posets, Proc. Amer. Math. Soc., 116 (1992), 1179_ 1181.

[B2] A. Björner, Topological methods in Combinatorics, In: Handbook of combinatorics (R. Graham, M. Grötschel, and L. Lovasz, eds.), Elsevier, New York, 1995.

[BGS] L. J. Billera, I. M. Gel'fand, and B. Sturmfels, Duality and minors of secondary polyhedra, J. Combin. Theory Ser. B, 57 (1993), 258-268.

[BKS] L. J. Billera, M. M. Kapranov, and B. Sturmfels, Cellular strings on polytopes, Proc. Amer. Math. Soc., 122 (1994), 549-555.

[BS] L. J. Billera and B. Sturmfels, Fiber polytopes, Ann. of Math. (2), 135 (1992), 527-549.

[G] B. Grünbaum, Convex Polytopes, Wiley, New York, 1967.

[GKZ] I. M. Gelfand, M. M. Kapranov, and A. V. Zelevinsky, Discriminants, Resultants, and MultiDimensional Determinants, Birkhäuser, Boston, 1994.

[L] C. L. Lawson, Software for $C^{1}$-interpolation, In: Mathematical Software III (J. Rice, ed.), Academic Press, New York, 1977.

[OR1] J. O'Rourke, Art Gallery Theorems and Algorithms, Oxford University Press, New York, 1987. 
[OR2] J. O'Rourke, Computational geometry column 18, Internat. J. Comput. Geom. Appl., 3 (1993), 107113; also in SIGACT News, 24:1 (1993), 20-25.

[RZ] J. Rambau and G. Ziegler, Projections of polytopes and the generalized Baues conjecture, Discrete Comput. Geom., 16 (1996), 215-237.

[SZ] B. Sturmfels and G. Ziegler, Extension spaces of oriented matroids, Discrete Comput. Geom., 10 (1993), 23-45.

[Z] G. Ziegler, Lectures on Polytopes, Graduate Texts in Mathematics, vol. 152, Springer-Verlag, New York, 1995.

Received January 23, 1997, and in revised form June 20, 1997. 